\title{
Dynamic gain decomposition reveals functional effects of dendrites, ion channels and input statistics in ultrafast population encoding
}

\author{
Chenfei Zhang ${ }^{1,2,3}$, Omer Revah ${ }^{4}$, Fred Wolf ${ }^{1,2,3,5,6,7}$, Andreas Neef ${ }^{1,2,3,5,6^{*}}$
}

1 Max Planck Institute for Dynamics and Self-Organization, Göttingen, Germany 2 Göttingen Campus Institute for Dynamics of Biological Networks, Göttingen, Germany

3 Bernstein Center for Computational Neuroscience, Göttingen, Germany

4 Koret School of Veterinary Medicine, Hebrew University of Jerusalem, Rehovot, Israel

5 Institute for the Dynamics of Complex Systems, University of Göttingen, Göttingen, Germany

6 Max Planck Institute of Experimental Medicine, Göttingen, Germany

7 Center for Biostructural Imaging of Neurodegeneration, Göttingen, Germany

\begin{abstract}
Understanding changes in information processing between different brain states is a major challenge for contemporary neuroscience. On the circuit level, the encoding capability is quantified by the spectrally resolved dynamic gain function. Its shape, in particular its bandwidth, is important for behavioral performance and possibly even mental capabilities. It has been presumed to be under evolutionary pressure, yet its underlying physiological determinants remain unclear. A systematic characterization had so far been precluded by the computational complexity of realistic neurons. Here we develop a general analysis framework applicable to neuron models as well as experiments on real neurons. We decompose the dynamic gain function by breaking down the physical signal transformation into subthreshold processes, namely current-to-voltage filtering and AP pattern formation, and suprathreshold action potential initiation. Applying this decomposition, we show that: 1 . Ultrafast population encoding can be realized by a type II neuron with Hodgkin-Huxley type potassium and sodium channels. 2. Adding a big dendrite improves high bandwidth encoding, primarily by attenuating low-frequency signal representation in the subthreshold region. 3. The initiation dynamics limits the $300-400 \mathrm{~Hz}$ bandwidth of real neurons, but not the $100 \mathrm{~Hz}$ bandwidth of the model. Our dynamic gain analysis can be universally applied to understand how (patho-) physiological modulation or mutagenesis changes a population's encoding capability.
\end{abstract}




\section{Introduction}

Vertebrate brains process information fast, within tenths of seconds 1]. In this process, large populations of neurons encode a common feed-forward input through changes in the population firing rate $\left(\mathrm{Fig} 1 \mathrm{~A} \mathrm{~A}_{1}\right)$. Although individual neurons fire irregularly, driven mainly by background input from weakly-correlated recurrent connections 2], their joint population firing rate faithfully represents the common input with millisecond precision 3]. This population encoding is quantified with the population's impulse response function, relating changes of the common input to changes of the population firing 4]. Its frequency domain representation is called the dynamic gain function $G(f)$, which displays a high bandwidth in cortical neurons (Fig $1 \mathrm{~A}_{2}$, data from 5]). Since in most situations, it is not known which common input is represented through which large population, a typical measurement of $G(f)$ uses abstractions and instead registers the precise timing of thousands of action potentials (APs), fired by a single neuron in response to an exogenous, fluctuating input. The statistics of the background input resembles the fluctuating stimuli neurons receive in vivo. In this way, the dynamic gain $G(f)$ is determined not through the activities of hundreds or thousands of neurons in response to a single common input, but through thousands of APs recorded from several similar neurons, one at a time, or obtained from a neuron model 6-11]. Measurements obtained from the same cell type in vivo and in vitro show similar encoding properties [3, demonstrating that the dynamic gain characterization ex vivo offers insights into the information encoding capabilities of populations in vivo.

Dynamic gain measurements are ideally suited to study cortical neurons, because they characterize neurons in a working regime resembling in vivo activity, measure a performance that is relevant on the systems level, and reveal surprising cellular characteristics otherwise hidden 5, 10. Almost all vertebrate neuron types studied so far can realize high bandwidth encoding (pyramidal cells from cortex L2/3 and L5, hippocampus CA1, purkinje cells, firing rate-adapting interneurons and fast spiking interneurons $3,6-10,12,17$, but not layer 1 interneurons [18]). This ability of ultrafast encoding is likely an important determinant of the brain's performance, and has been previously suggested to underlie evolutionary pressure 9]. Therefore, the underlying mechanisms are of interest, and theoretical studies suggested that two candidate mechanisms play a major role. One is the active intrinsic AP initiation dynamics 19 24, mainly determined by the sodium channel voltage sensitivity. A high voltage sensitivity allows for rapid AP initiation and thereby improves AP timing precision, which further enhances the encoding ability in the high frequency region. The other mechanism is the passive morphology, including the position of the initiation site 25, 26, and the dendrite size [11, 15, 27. It has been suggested that neuron morphology can also accelerate AP initiation, seen at the soma 25], or in the axon [1].

Despite these findings and the presumed importance of ultrafast population encoding, its biophysical origins and specific parameter requirements are still under debate, not least because experimental observations paint a more complex picture than the theoretical studies. The fact that human pyramidal neurons and mouse fast spiking interneurons, two cell types with vastly different dendrite sizes and ion channel types, both achieve cutoff frequencies above $500 \mathrm{~Hz}$ 10, 14, suggesting that a range of neuron properties can support this performance. The relative contributions of dendrite size and axonal conductances have not been studied systematically (but see 26]), mostly because it is not clear how different conditions or different models can be compared in an unbiased way. Such comparisons are not trivial, because a neuron does not have a single dynamic gain curve but rather a whole family of curves. The shape of the dynamic gain curve can drastically change depending on the neuron's working point, determined by the input fluctuation amplitude and correlation time, the firing rate and firing pattern (e.g. 10 28]). In some of our recent experimental and theoretical studies, we determined the working points by fixing the firing rate and the coefficient of variation of inter-spike intervals 
$C V_{I S I} 926$ (see 29 for theory). In others, we limited the range of subthreshold voltage fluctuations [3], or used a mixture of both criteria [5]. However, a consistent framework for the choice of working points is currently missing and it is not clear if and how this choice impacts the observed contributions of ion channel properties or morphology on the dynamic gain curve.

Here we introduce an analysis framework that guides not only the choice of working points but also allows for a detailed attribution of dynamic gain features in experimental and simulation data. Our dynamic gain decomposition simply breaks down the transformation of input currents into spike times, by following the physical signal transformation and largely separating subthreshold processes from threshold dynamics. In doing so, we relate the theoretical construct of dynamic gain as the population's signal transfer function to the biophysical mechanisms that shape it. This makes it possible to interpret dynamic gain changes, and we exemplify this not only for simulated data, but also for recordings from cortical pyramidal neurons. When applied to a biophysically plausible, multi-compartment model with Hodgkin-Huxley type potassium and sodium channels, we find that the high bandwidth encoding is due to its type II excitability. A type I model counterpart fails to reproduce ultrafast population encoding. The addition of a dendrite impacts the dynamic gain primarily by shaping the impedance and only modulates the bandwidth that is determined by the excitability type. Interestingly, we find that the AP initiation dynamics limits the bandwidth in the experimental data, but not the model. 


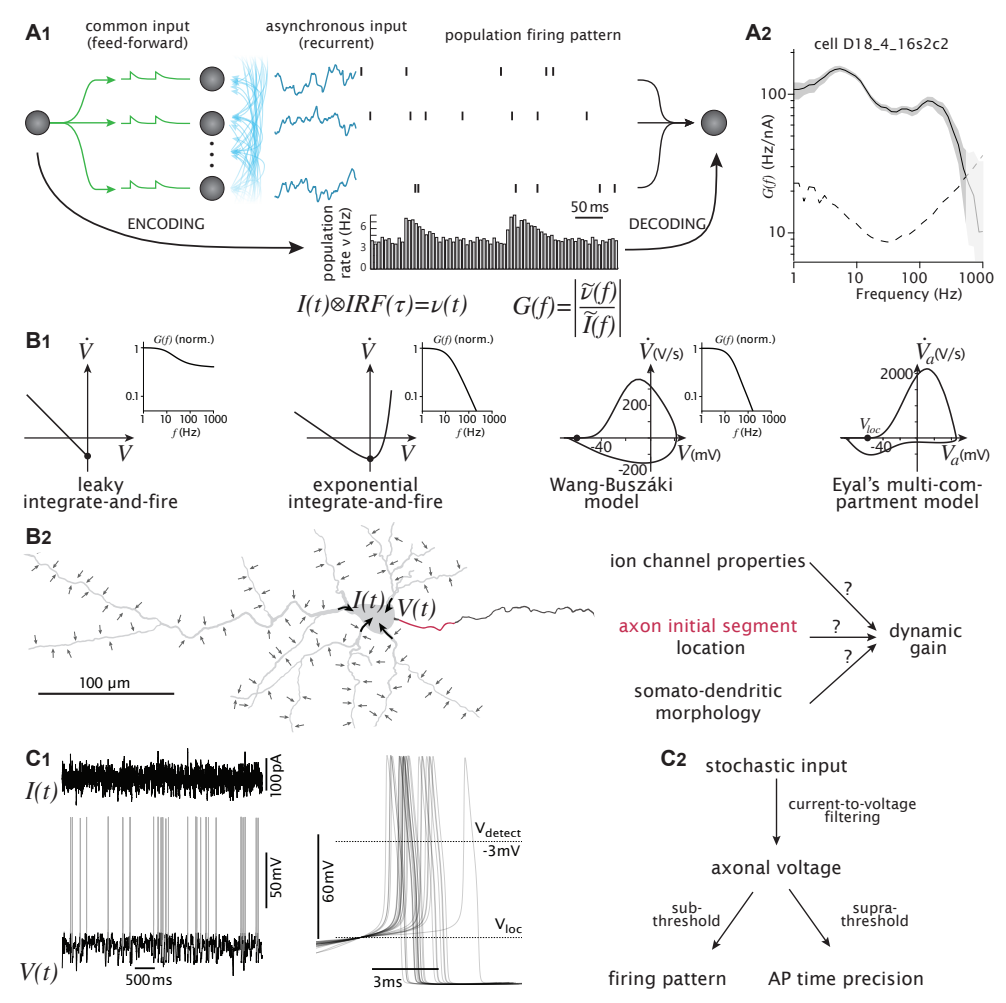

Figure 1: An introduction to dynamic gain and a concept formulation of deriving dynamic gain decomposition. A1 A population encoding scheme. Feedforward input (green) diverges onto a recurrently connected neuronal population. The dominating, asynchronous, recurrent input causes weakly-correlated AP patterns. Nevertheless, the population rate can faithfully reflect changes in the common input. A downstream neuron can detect this signal, filtered through the population's transfer function, the dynamic gain $G(f)$. A2 Experimentally determined dynamic gain function of a layer 5 pyramidal cell, obtained from 2,330 APs (data from [5]). Note the the wide bandwidth, and the maxima and minima. Confidence interval (grey band) and noise floor (dashed) are obtained by bootstrapping (see Models and Methods). B1 Evolution of neuron models' phase plots, from point neurons without initiation dynamics to Eyal's multi-compartment model. Locations of local minima during AP initiation are marked with dots and denoted as $V_{l o c}$. Normalized dynamic gain functions of the first three model variants are shown next to the phaseplots. B2 Schematic representation of neuronal signal transformation. Distributed synaptic inputs drive current into the soma and axon; the axonal voltage $\left(V_{a}\right)$ changes. For real neurons and biophysically plausible multi-compartment models, various parameters shape these transformations, attribution of dynamic gain changes to specific parameters is a challenge. C1 Globally, Eyal's model displays irregular firing patterns in response to stochastic stimuli. Locally, at AP onset, the initiation dynamics varies, signified by the large spread in the delay between the last positive crossing of $V_{l o c}$ and the AP detection voltage $(0 \mathrm{mV})$. C2 Decomposition of AP initiation process. The transformation of somatic input into $V_{a}$ fluctuations at the AP initiation site can be idealized as an effective impedance. $V_{a}(t)$ contains the information of firing pattern (time intervals when $V_{l o c}$ can be reached) and the precise, sub-millisecond AP timing, respectively determined by the subthreshold dynamics before $V_{l o c}$ and suprathreshold dynamics afterwards. This decomposition can disentangle the functional effects of neuronal properties on population encoding. 


\section{Models and Methods}

Neuron morphology and biophysical properties We used the multicompartment model studied in 11. The neuron model is composed of a dendrite, a soma and an axon (see supplementary Fig S1), and studied at the same time resolution of $25 \mu \mathrm{s}$. All three compartments are cylinders. The soma has a diameter of $20 \mu \mathrm{m}$ and a length of $30 \mu \mathrm{m}$. The axon is composed of an axon initial segment (AIS) with a diameter of $1 \mu \mathrm{m}$ and a length of $50 \mu \mathrm{m}$, and a myelinated axon with a diameter of $1 \mu \mathrm{m}$ and a length of $1000 \mu \mathrm{m}$. To examine the impact of the dendrite on population response, we studied three model variants with different dendrite sizes used in 11. The first model is without the dendrite, the second model has a dendrite with a diameter of $3 \mu \mathrm{m}$ and a length of $2324 \mu \mathrm{m}$, and the third model has a dendrite with a diameter of $5 \mu \mathrm{m}$ and a length of $3000 \mu \mathrm{m}$ (these models are identified in 11] by axonal loads $\rho_{\text {axon }}$ of 12,95 and 190). The dendrite, soma, and AIS, contain voltage dependent ion channels, the myelinated axon is passive. The axial resistance $R_{a}$ is $100 \Omega \mathrm{cm}$, the reversal potential for the leak current $V_{L}$ is $-70 \mathrm{mV}$, the specific membrane capacitance $c_{m}$ is $0.75 \mu \mathrm{F} / \mathrm{cm}^{2}$ and the specific leak conductance $g_{L}$ is $3.3 \times 10^{-5} \mathrm{~S} / \mathrm{cm}^{2}$, except for the myelinated axon, where the last two parameters are reduced to $0.02 \mu \mathrm{F} / \mathrm{cm}^{2}$ and $6.6 \times 10^{-7} \mathrm{~S} / \mathrm{cm}^{2}$. Sodium and potassium channels are distributed in the active part of the neuron model. The sodium current is described by $I_{N a}=\bar{g}_{N a} m^{3} h\left(V-E_{N a}\right)$ with $E_{N a}$ set to $50 \mathrm{mV}$. The slope factor $q_{a}$ of the stationary gating variable $m_{\infty}$ is $9 \mathrm{mV} . \bar{g}_{N a}$ is $20 \mathrm{pS} / \mu \mathrm{m}^{2}$ in the dendrite, $800 \mathrm{pS} / \mu \mathrm{m}^{2}$ in the soma, and $8000 \mathrm{pS} / \mu \mathrm{m}^{2}$ in the AIS. The potassium current is described by $I_{K}=\bar{g}_{K} n\left(V-E_{K}\right)$ with $E_{K}$ set to $-85 \mathrm{mV} . \bar{g}_{K}$ is $10 \mathrm{pS} / \mu \mathrm{m}^{2}$ in the dendrite, $320 \mathrm{pS} / \mu \mathrm{m}^{2}$ in the soma, and 1500 $\mathrm{pS} / \mu \mathrm{m}^{2}$ in the AIS. The dynamics of gating variables are adapted from 30 with the model temperature set to $37^{\circ} \mathrm{C}$. All simulations were performed with NEURON 7.6 and 8.0 31] compiled with Python 3.7 and 3.9. The code is available on github: https://github.com/chenfeizhang/Code_Eyal_gwdg

Neuron model simulation and dynamic gain function The neuron model was driven by a current stimulus injected to the middle of the soma. To create the AP phase plots and measure the AP initiation speed, the stimulus was a constant current just above rheobase. We recorded the voltage in the AIS, 47 $\mu \mathrm{m}$ away from the soma, during the first AP. When comparing the AP initiation speed of different model variants, the local minima of the phase plots were shifted to $0 \mathrm{mV}$ in the $\mathrm{x}$ axis.

The encoding ability of the neuron population can be evaluated by injecting each neuron with an independent realization of the background noise combined with a small sinusoidal signal:

$$
I(t)=I_{0}+A \sin (2 \pi f t)+I_{\text {noise }}(t)
$$

Here $I_{0}$ is the mean input, $A$ is the amplitude of the sinusoidal signal, and $I_{\text {noise }}$ is a zero-mean stochastic stimulus generated by the Ornstein-Uhlenbeck (OU) process:

$$
\tau d I_{\text {noise }}(t)=-I_{\text {noise }}(t) d t+\sqrt{2 \tau} \sigma_{I} d W(t)
$$

where $\tau$ is the correlation time, $\sigma_{I}$ is the standard deviation (std), and $W(t)$ is a Wiener process with zero mean and unit variance. The population firing rate can be expanded as:

$$
\nu(t)=\nu_{0}+G(f) \cdot A \sin (2 \pi f t+\phi(f))+\mathcal{O}\left(A^{2}\right)
$$

where $\nu_{0}$ is the mean firing rate, $G(f)$ is the tuning ratio between the output population firing rate and the sinusoidal signal at frequency $f, \phi(f)$ is the phase shift dependent on the frequency, and $\mathcal{O}\left(A^{2}\right)$ is the higher order term of the output. We name the linear response part of the population firing $G(\cdot)$ as the dynamic gain function. The dynamic gain could be theoretically calculated in a straight 
forward way as the ratio of the Fourier transform of the spike output, and the Fourier transform of the current input.

$$
G(f)=\left|\frac{\mathcal{F}(\nu(t))}{\mathcal{F}(I(t))}\right|
$$

The firing rate can be understood as a sum of delta functions, located at the AP times $t_{i}$ :

$$
\nu(t)=\sum_{i=1}^{n} \delta\left(t-t_{i}\right)
$$

Here, we used the Fourier transform method proposed by 8 :

$$
G(f)=\left|\frac{\mathcal{F}\left(C_{I \nu}\right)}{\mathcal{F}\left(C_{I I}\right)}\right|=\frac{\langle\nu\rangle \cdot|\overline{\mathcal{F}(S T A)}|}{\frac{2 \tau \sigma_{I}^{2}}{1+(2 \pi \tau f)^{2}}}
$$

$\mathcal{F}\left(C_{I \nu}\right)$ is the Fourier transform of input-output cross-correlation. It is equal to the multiplication of the mean firing rate $\langle\nu\rangle$ and the complex conjugate of the Fourier transform of the spike-triggered average current, i.e. $\overline{\mathcal{F}(S T A)}$. To calculate the spike-triggered average input, AP times were defined as the time points at which the detection voltage was crossed from below. The detection voltage was chosen as the voltage at which the $\mathrm{AP}$ waveform reaches its maximal slope. $\mathcal{F}\left(C_{I I}\right)$ is the Fourier transform of the input auto-correlation function. Because this method does not require a sinusoidal stimulus component, the stimulus is the OU process defined above. Its power spectral density is known, and due to the Wiener-Khinchin theorem, it equals $\frac{2 \tau \sigma_{I}^{2}}{1+(2 \pi \tau f)^{2}}$.

For each dynamic gain function, we generated 400 trials of 1000 seconds. Each trial returns a 1s long STA based on approximately 5000 APs. The average of these 400 STA is the final STA for calculating the dynamic gain function. To de-noise the dynamic gain in the high frequency region, we applied a collections of Gaussian filters to the Fourier transform components of the STA, and the widths of the Gaussian filters increase with the frequency [8]. To evaluate the bootstrap confidence interval of the dynamic gain functions, we re-sampled 400 STAs with replacement for 1000 times and recalculated the final STAs and corresponding gain curves. The central 95 percent are shown in our results, this confidence interval is often more narrow than the line widths. To determine the significance threshold of the gain curves, we shifted the AP times by a random interval, larger than 1 second. This shuffling was performed 100 times and the resulting gain curves were recalculated. The 95th percentile upper bound of those random gain curves forms the noise floor and we only show results above that noise floor. For a simple characterization of the shape of dynamic gain functions, we denote the cutoff frequency as the point where the dynamic gain drops below $70 \%$ of the dynamic gain value at $1 \mathrm{~Hz}$.

Dynamic gain decomposition The effective impedance describes, in a spectrally resolved manner, the transformation between the fluctuating current injected into the soma and the fluctuating voltage measured at the AP initiation site. To calculate it in simulations, we added a sinusoidal signal to the stochastic stimulus. The amplitude of the sinusoidal signal is one fifth of the std of the stimulus. For each sinusoidal frequency, we generated 20,000 seconds of axonal voltage traces. To remove the obviously non-linear AP waveforms, voltages above $-50 \mathrm{mV}$ are set to $-50 \mathrm{mV}$. When $\sigma_{I}$ is relatively small, i.e. when the neuron is operating close to mean-driven, it can happen that the spike-induced voltage excursions of after-hyperpolarization and after-depolarization exceed the inputdriven voltage fluctuations. In this case, voltage excursions towards the negative values, e.g. voltages below $-70 \mathrm{mV}$ are also replaced by a threshold value. We averaged 20,000 pieces of $1 \mathrm{~s}$ of axonal voltage traces, to obtain the sinusoidally modulated average voltage. The effective impedance at the sine frequency is 
the ratio of sinusoidal voltage modulation amplitude to the sinusoidal current modulation amplitude. We denote the effective impedance as $Z_{\text {eff }}(f)$. To analyze experimental data, voltage excursions above a data-specific threshold are clipped. $Z_{\text {eff }}$ is determined as the ratio between the Fourier transform of clipped voltage traces and the Fourier transform of current traces. The spike gain at frequency $f$ is the ratio of the dynamic gain function $G(f)$ to the effective impedance $Z_{\text {eff }}(f)$. It describes the encoding of axonal voltage fluctuations into spiking in a spectrally resolved manner, denoted as $G_{s p}(f)$ :

$$
G_{s p}(f)=G(f) / Z_{\text {eff }}(f)
$$

Typically, we detect APs at a voltage, where the rate of voltage rise is maximal. For Eyal's model without the dendrite, this corresponds to $-3 \mathrm{mV}$. For the data, it is typically between $-5 \mathrm{mV}$ and $+5 \mathrm{mV}$ and here we chose $0 \mathrm{mV}$ for the analysis of all experimental data. When we lower this $V_{\text {detect }}$, we obtain modified, earlier AP times. Importantly, we consider only the last positive crossings of $V_{\text {detect }}$ before the full AP depolarization, therefore, the number of APs does not change. When we calculate $G(f)$ with AP times obtained with a detection voltage around AP initiation threshold, we obtain the zero-delay dynamic gain $G^{0}(f)$. For Eyal's model, we obtained the phase plot of an AP fired just above rheobase input and used the voltage of the local minimum $V_{l o c}$ as an approximation of the AP initiation threshold. For experimental data, the corresponding $V_{l o c}$ can only be estimated. The ratio $G(f) / G^{0}(f)$ is the dynamic gain decay caused by the variable initiation dynamics.

Experimental data analysis We obtained the experimental data from 5 and used the dynamic gain decomposition to interpret encoding differences between individual neurons, and between the treatment group and the control group. The dynamic gain functions were calculated with the Fourier transformation method, using the spike triggered average current. We re-sampled the spike triggered current of all APs with replacement for 1000 times, then took the central 95 percent range as the bootstrapping confidence intervals. We performed the same spike time shuffling as in Eyal's model for 1000 times, then took the 95 percent upper bound as the noise floor. Decomposing the dynamic gain function into effective impedance, zero-delay spike gain and gain decay, we provided an interpretation on encoding variability of two neurons. On the group level, $G(f), Z_{\text {eff }}$, and $G_{s p}(f)$ were calculated for all 15 neurons of the treatment group and all 9 neurons of the control group. The grand average within groups was obtained for the frequency range in which all individual traces were significant, i.e. above the noise floor. For the statistical analysis of $G(200 H z)$, and $G_{s p}(200 H z)$, we tested for normality using Jarque-Bera tests and then tested for equal mean using t-tests. The phase plots for experimental data were obtained from the voltage traces by binning voltage data and then averaging the corresponding voltage derivative of each voltage point across all entries of each bin. The resulting average voltage derivative is plotted against the centre of the voltage bin.

\section{Results}

\section{Decomposing dynamic gain provides subcellular resolu- tion for the analysis dynamic population coding.}

Our decomposition of dynamic gain is motivated by the simplifications that underlie the lineage of frequently used neuron models $\left(\mathrm{Fig} 1 \mathrm{~B}_{1}\right)$. The simplest of these models, the leaky integrate-and-fire (LIF) neuron, abstracts all subthreshold dynamics as a low-pass filter governed by the membrane time constant, and all suprathreshold dynamics as instantaneous. Its dynamic gain function $G(f)$ can be interpreted as a concatenation of a current-to-voltage transformation, captured by 
the impedance $Z(f)$, and a voltage-to-firing rate transformation, the spike gain $G_{s p}(f)$. Rewriting the definition of the dynamic gain, we have:

$$
G(f)=\left|\frac{\mathcal{F}(\nu)}{\mathcal{F}(I)}\right|=\left|\frac{\mathcal{F}(V)}{\mathcal{F}(I)}\right| \cdot\left|\frac{\mathcal{F}(\nu)}{\mathcal{F}(V)}\right|=Z(f) \cdot G_{s p}(f)
$$

, where $G_{s p}(f)$ represents the firing frequency preference hidden in the firing pattern.

The exponential integrate-and-fire (EIF) model adds a suprathreshold AP initiation dynamics. Once the membrane voltage passes the local minimum of the phase plot at $V_{l o c}$, the voltage derivative $d V / d t$ increases exponentially with voltage $\left(\mathrm{Fig} 1 \mathrm{~B}_{1}\right)$. Between passing $V_{l o c}$ and reaching an AP detection threshold $V_{\text {detect }}$, the dynamics is determined by a mixture of the intrinsic AP initiation current and the extrinsic stochastic stimulus, leading to variable initiation delays (see example in Fig $1 \mathrm{C}_{1}$ ). Adding the intrinsic dynamics drastically changes the high frequency limit of $G(f)$ from a constant value for LIF to a power law decay for EIF $\left(\mathrm{Fig} 1 \mathrm{~B}_{1}\right)$ 19,20]. The simple structure of the EIF model allowed Fourcaud-Trocmé and colleagues to relate the voltage dependence of the initiation current to the bandwidth of $G(f)$. Here we propose to study this relation with a simple, phenomenological approach that is readily generalized to models of arbitrary complexity. When we lower $V_{\text {detect }}$ towards $V_{l o c}$, the influence of the suprathreshold dynamics is minimized and we converge to the behavior of a LIF-like model with a hard threshold and a flat gain curve. Therefore, the gain decay of the EIF model is closely associated with the suprathreshold dynamics after $V_{l o c}$. In the general case, we obtain this gain decay as the ratio of two dynamic gain curves, $\frac{G^{0}(f)}{G(f)}$, where the 'zero-delay' dynamic gain $G^{0}(f)$ is obtained with 'zero-delay' APs detected already at $V_{l o c}$, while $G(f)$ is obtained with the conventional, much more depolarized AP detection threshold. In other words, we relate the encoding capability of a LIF-like model version to that of the full model and capture the result in the spectrally resolved gain decay.

The introduction of other ion channel types marks the next level of model complexity, represented by conductance-based models, such as the Wang-Buszáki model 32]. These additional dynamical variables enable AP repolarization and richer neuronal dynamics, introducing different dynamical bifurcations at the AP threshold. This allows for a variety of firing patterns and consequently for different spike gain shapes. In spatially extended multi-compartment models (e.g. Eyal's model [11]), currents and voltage gradients between compartments increase the complexity even further. The presence of various ion channels together with the extended morphology results in a more complex current-to-voltage transformation that we describe with an effective impedance $Z_{\text {eff }}(f)$, which also includes the stimulus filtering along the path from the somatic stimulus source to the axonal AP initiation site.

In summary, our decomposition approach establishes three $G(f)$ components: 1) effective impedance $\left.Z_{\text {eff }}(f), 2\right)$ zero-delay spike gain $G_{s p}^{0}(f)$ and 3) dynamic gain decay $\frac{G^{0}(f)}{G(f)}$. The first two capture subthreshold influences on $G(f)$, the third captures the impact of AP initiation dynamics (Fig $1 \mathrm{C}_{2}$ ). Dynamic gain is the product of these transformations:

$$
G(f)=Z_{\text {eff }}(f) \cdot G_{s p}^{0}(f) \cdot \frac{G(f)}{G^{0}(f)} .
$$

For such a decomposition, we only require the waveforms of stimulus and membrane voltage (Models and Methods). It can therefore be performed on neuron models of arbitrary complexity and even on recordings from real neurons $\left(\mathrm{Fig} 1 \mathrm{~B}_{2}\right)$. In the next sections, we will analyze the functional effects of biophysical and morphological parameters using experimental data and different variants of Eyal's model, which feature a relatively wide encoding bandwidth 11. But first, Eyal's model will be characterized and studied at different working points. 


\section{Population encoding performance depends on neuron ex- citability and working point.}
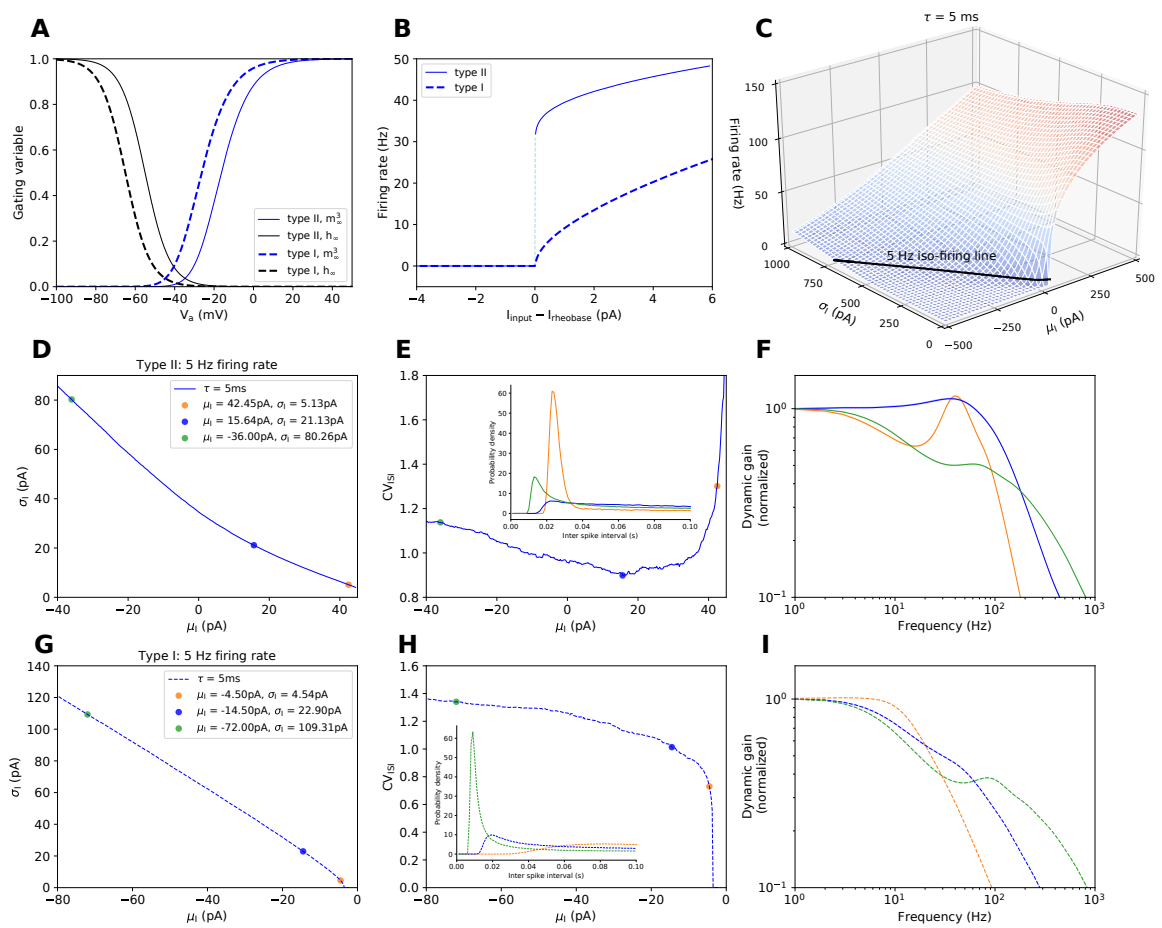

I

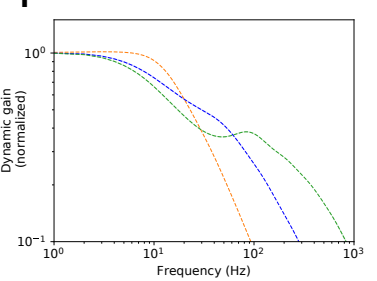

Figure 2: Eyal's model can realize high bandwidth encoding only with a type II, but not a type I excitability. A To switch the excitability of Eyal's model from type II to type I, the voltage dependence of sodium channel activation and inactivation is shifted $10 \mathrm{mV}$ towards more hyperpolarized potentials. $V_{a}$ denotes the voltage at the AP initiation site. B F-I curves with the rheobase thresholds aligned to $0 \mathrm{pA}$. C 2-D firing rate surface of Eyal's type II model as a function of mean and std of stochastic stimulus ( $\tau=5 \mathrm{~ms}$ ). The $5 \mathrm{~Hz}$ iso-firing rate line is labelled black. D $\sigma_{I}-\mu_{I}$ and $\mathbf{E} C V_{I S I^{-}} \mu_{I}$ relation of the type II model at $5 \mathrm{~Hz}$ firing rate. Colored dots indicate three example working points, ranging from nearly mean-driven (orange) to fluctuation-driven (blue), and to very fluctuation-driven (green). Corresponding ISI distributions are shown in the inset panel. F Dynamic gain functions of the three example working points. High bandwidth encoding is realized only when the neuron model is fluctuation-driven (blue). G through $\mathbf{I}$ as D through F, but for the type I model. I The type I model does not realize high bandwidth encoding. Note, that an increased $\sigma_{I}$ enhances the dynamic gain in the high frequency region, however, the cutoff frequency always remains below $16 \mathrm{~Hz}$.

We first examined the dynamical properties of the multi-compartment model characterized by Eyal and colleagues [11], specifically the neuron model consisting of only a soma and an axon but no dendrite. For comparison, we devised a model variant that shares its morphology and is equipped with the same ion channel types at the same densities and with the same voltage sensitivities. However, in the new model variant, sodium channels activate and inactivate with a $10 \mathrm{mV}$ shift towards more hyperpolarized potentials (Fig 2 A). In response to various constant currents injected into the soma, the firing rate $\nu$ of the original model displays a large discontinuity of about $32 \mathrm{~Hz}$ upon reaching the rheobase, indicating that it is a type II model. Its model variant, however, has a continuous F-I curve, indicating 
it is a type I model (Fig $2 \mathrm{~B}$, rheobase currents aligned at $0 \mathrm{pA})$.

We next examined the models' response to stochastic input with a correlation time $\tau=5 \mathrm{~ms}$. Given that the firing rate has a strong impact on the bandwidth of population response 20, the firing rate is often fixed when different models or stimulus conditions are compared (e.g [33]). Here, probing the two-dimensional firing rate surface spanned by stimulus mean $\mu_{I}$ and standard deviation $\sigma_{I}$, we identified the iso-firing rate line at $5 \mathrm{~Hz}(\mathrm{Fig} 2 \mathrm{2})$. Along this line, the neuron's firing regime changes from nearly mean-driven to fluctuation-driven. The curvature of the iso- $5 \mathrm{~Hz}$ curve in the $\sigma_{I^{-}}-\mu_{I}$ plane differs between the two excitability types. It is slightly concave for type I, and slightly convex for type II excitability (Fig $2 \mathrm{P}$ and $2 \mathrm{G}$ ). The firing irregularity, quantified by the coefficient of variation of inter-spike intervals $C V_{I S I}$, differs more noticeably between the model variants. While the type I model's firing irregularity increases monotonously with $\sigma_{I}(\mathrm{Fig} 2 \mathrm{H})$, the type II model displays a minimum $C V_{I S I}$ for intermediate $\sigma_{I}$ values. Towards more mean-driven conditions, $C V_{I S I}$ increases strongly (Fig $2 \mathrm{E}$ ), because the intrinsic firing rate of $32 \mathrm{~Hz}$ is much higher than the $5 \mathrm{~Hz}$ target rate. In the presence of small input fluctuations around a near-rheobase average input, this low target rate is realized by bursts of near-regular rapid firing separated by long intervals. This is qualitatively very different from the irregular firing produced at working points further away from the mean-driven regime. For both model variants, we chose three working points, i.e. $\mu_{I}-\sigma_{I}$ combinations, covering the range from mean-driven to strongly fluctuation driven (colored dots in Fig 2D and G). The inter-spike interval (ISI) distributions at those working points (insets in Fig $2 \mathrm{E}$ and $\mathrm{H}$ ) further illustrate the very regular firing pattern of the type II model close to the mean-driven condition. As the input fluctuations increase, the ISI distributions become more similar.

When we calculated the dynamic gain functions for the two models at the respective working points, the results were again comparable for the strongly fluctuation-driven cases, but strikingly different for other working points. When the original type II model is mean-driven $\left(\sigma_{I}=5.13 \mathrm{pA}\right)$, the dynamic gain function appears to have a low bandwidth, dropping to $70 \%$ magnitude already at $10 \mathrm{~Hz}$ (orange in Fig $2 \mathrm{~F}$ ). At intermediate and high frequencies, the shape of the dynamic gain function is dominated by a strong resonance around $40 \mathrm{~Hz}$, which mirrors the peak in the ISI distribution around $25 \mathrm{~ms}$. Increasing $\sigma_{I}$ to $21.13 \mathrm{pA}$ significantly broadens the resonance peak and increases the cutoff frequency of the dynamic gain function, which now resembles the gain curve shown in 11. (blue line). The corresponding ISI distribution is also substantially flattened, indicating the transition from mean-driven to fluctuation-driven. Interestingly, a further increase to $\sigma_{I}=80.26 \mathrm{pA}$, does not enhance the population encoding ability further (green line). Instead, at this very fluctuation-driven working point, the dynamic gain function is dominated by a low-pass filter in the low frequency region with an apparent cutoff frequency of around $12 \mathrm{~Hz}$. Towards higher frequencies, a shoulder appears and results in a local maximum around $60 \mathrm{~Hz}$. The dynamic gain in the high frequency region exceeds the other two gain curves. In parallel, the ISI distribution narrows again and moves closer to zero. The most probable ISI now is approximately $70 \mathrm{~Hz}$, corresponding to the shoulder in the dynamic gain function. These results demonstrate that the encoding ability critically depends on the working point; the high encoding bandwidth reported for this type II model, is realized only in a fluctuation-driven regime with intermediate $\sigma_{I}$.

The dynamic gain curves of the type I model behave strikingly different. None of the three working points lead to high bandwidth encoding (Fig 21). When mean-driven $\left(\sigma_{I}=4.54 \mathrm{pA}\right)$, the dynamic gain function has a cutoff frequency below $30 \mathrm{~Hz}$, and decays with a slope of -1 in the log-log scale (orange dashed line), similar to the exponential integrate-and-fire model with standard AP initiation dynamics 20. Increasing $\sigma_{I}$ to $22.90 \mathrm{pA}$ to reach the fluctuation driven regime, the dynamic gain is enhanced in the high frequency region, while the cutoff frequency becomes smaller (blue dashed line). Further increasing $\sigma_{I}$ to $109.31 \mathrm{pA}$ leads to a plateau in the low frequency region with a even lower cutoff frequency (green 
dash line). The dynamic gain function is larger in the high frequency with a shoulder around $100 \mathrm{~Hz}$, similar to the type II model's gain curve at the very fluctuation-driven working point (compare two green curves in F and I). Again, the ISI distribution features a peak around the time interval that corresponds to the shoulder's frequency range. These results demonstrate that the type I model cannot reproduce high frequency encoding throughout the biophysically plausible range of working points. In summary, we found that the encoding bandwidth can be increased tenfold, not by manipulating morphology or ion channel voltage sensitivity, but simply by increasing the potassium current around threshold to achieve a type II excitability. We next use the dynamic gain decomposition to investigate how this occurs.

\section{AP initiation dynamics does not limit the encoding band- width of Eyal's model.}

Before we decompose the models' dynamic gains curves as proposed above, we first take a closer look at the AP initiation dynamics. We previously demonstrated that high bandwidth encoding is closely associated with the voltage sensitivity of intrinsic AP initiation dynamics, especially in the voltage region where it rises from the local minimum of the phase plot 26]. Using the voltage $V_{a}$, recorded at the AP initiation site, we compared the phase plots of type I and type II models with their local minima $\left(V_{l o c}\right)$ aligned at $0 \mathrm{mV}(\mathrm{Fig} 3 \mathrm{~A})$. Although the voltage sensitivity of the sodium channel dynamics is identical for the two model variants, the voltage derivative of the type II model rises substantially more quickly out of $V_{l o c}$. The slower initiation of the type I model is caused by its $16 \mathrm{mV}$ more hyperpolarized $V_{l o c}$. The sodium channels' voltage dependence was shifted by only $10 \mathrm{mV}$, meaning that AP initiation proceeds at a voltage range with fewer activated sodium channels and of course even less potassium channels. As a consequence, the type I model's AP initiation current displays a weaker voltage sensitivity.

When we gradually lowered the AP detection voltage $V_{\text {detect }}$ down to $V_{l o c}$, we obtained new, earlier AP times, but the number of APs stays constant because we considered only those last positive crossings of $V_{\text {detect }}$ that lead to fully developed APs. With these redefined AP times, we recalculated the dynamic gain function (Fig $3 \mathrm{~B}$, type II model for demonstration). As we detect the APs earlier, we limit the voltage range, across which the initiation dynamics can influence the encoding of inputs. On the other hand, this also limits the time and voltage interval during which the extrinsic stimulus can impact the precise AP time. Comparing the dynamic gain functions for two detection thresholds, we can relate their differences to the AP dynamics in the voltage interval between $V_{\text {detect }}^{1}$ and $V_{\text {detect }}^{2}$. We find that lowering $V_{\text {detect }}$ all the way from $-3 \mathrm{mV}$ down to $-48 \mathrm{mV}$ has very limited impact on the dynamic gain function. It only slightly enhances the encoding at large frequencies (above $500 \mathrm{~Hz}$ ). However, a further decrease of $V_{\text {detect }}$ by $5 \mathrm{mV}$ or $10 \mathrm{mV}$ drastically improves high frequency encoding and has considerable effects at frequencies down to $50 \mathrm{~Hz}$, but not below. These results demonstrate that the AP initiation dynamics of the type II model is the main determinant for the strong decay of dynamic gain functions above $50 \mathrm{~Hz}$. When the detection voltage reaches $V_{l o c}$, the AP initiation dynamics after $V_{l o c}$ is ignored. The resulting neuron model is similar to a LIF model with a hard threshold at $V_{l o c}$, and indeed, the ensuing zero-delay dynamic gain function becomes almost flat in the high frequency region as expected for a LIF model (continuous light blue curve in Fig $3 \mathrm{~B}$ ).

The subthreshold dynamics, is still reflected in zero-delay dynamic gain. It governs the transfer of current to membrane voltage and thereby determines during which time intervals, APs can appear at all. Further decreasing the detection threshold cuts into the subthreshold dynamics. The resulting changes of AP times can be substantial and affect the firing pattern and thereby also the dynamic gain in lower frequency regions (light blue dashed line in B). We can conclude that the type II model's near constant encoding capability between 5 and $50 \mathrm{~Hz}$, does not 
originated from its fast AP initiation after $V_{l o c}$. Instead, it is determined by the subthreshold dynamics before $V_{l o c}$.

Even the type I model's slower AP initiation dynamics does not limit encoding between 5 and $50 \mathrm{~Hz}$ frequency for the fluctuation driven regimes (blue and green). This is evident from the results of the gain decay analysis applied to the type I model (supplementary Fig S2). It is reflected in Fig 3E, indicating that only in the nearly mean-driven regime (orange), the bandwidth of the gain decay limits the overall dynamic gain bandwidth.

To quantify the voltage trajectories' variability during AP initiation, we determined the time required for each $\mathrm{AP}$, to progress from the last positive crossing of $V_{l o c}$ to $V_{\text {detect }}=-3 \mathrm{mV}$ (see Models and Methods). This AP initiation delay is a random variable. Its statistics is determined by the interplay between intrinsic AP initiation dynamics and extrinsic stimulus fluctuations. The initiation delay's variance originates mainly from very variable dynamics very close to $V_{l o c}$, where the intrinsic currents are lowest and initiation proceeds slowest. For the type II model, $90 \%$ of the total initiation delay are spent on crossing the first $10 \mathrm{mV}$ after $V_{l o c}$, even though they represent only $18 \%$ of the voltage interval. These $10 \mathrm{mV}$ also contribute $99 \%$ of the delay's standard deviation. Fig $3 \mathrm{~B}$ illustrates that these first $10 \mathrm{mV}$, from -58 to $-48 \mathrm{mV}$, also account for the bulk of the dynamic gain decay associated with the AP initiation delay. We can use the dynamic gain decay $\frac{G(f)}{G^{0}(f)}(\mathrm{Fig} 3 \mathrm{E})$, together with the distributions of AP initiation delay, to quantify the impact of AP initiation dynamics on high frequency encoding at the three working points in Fig $2 \mathrm{~F}$ and I. For the type II model, when the activity is mean-driven (orange), the AP initiation delay distribution is relatively flat with a most probable delay as large as $4 \mathrm{~ms}$. Increasing $\sigma_{I}$ reduces the mean of the distribution towards $0 \mathrm{~ms}$ (blue and green), indicating that the AP initiation delay is no longer limited by the intrinsic AP initiation dynamics. Instead, larger depolarizing inputs increase $d V / d t$, and accelerate the escape from the slow AP initiation region near $V_{l o c}$. This faster initiation is paralleled by a rightward shift in the dynamic gain decay. The bandwidth related to the initiation dynamics increases more than three-fold. For the type I model, similar transitions of the dynamic gain decays and AP initiation delay distributions can be observed when increasing $\sigma_{I}$. While the two model types behave similarly when driven with very large $\sigma_{I}$, towards the mean-driven working points the type I model's AP initiation delay distribution is substantially more flattened, and consequently the dynamic gain decay has a much lower bandwidth. We attribute this to the different firing patterns close to the mean-driven condition. The type I model produces near-regular firing without the high-frequency bursts of its type II relative. Therefore, the average AP initiation delay would approach $200 \mathrm{~ms}$ when $\sigma_{I}$ decreases to zero and the firing rate is kept at $5 \mathrm{~Hz}$. For such initiation delays exceeding the input correlation time, the input fluctuations can cause particularly large variability and consequentially deteriorate encoding precision.

\section{Factoring membrane potential susceptibility uncovers the dependence of encoding bandwidth on active subthresh- old conductances and firing pattern preference.}

The subthreshold part of the encoding process, which is described by the zerodelay dynamic gain $G^{0}(f)$, can be further decomposed into effective impedance $Z_{\text {eff }}(f)$ and zero-delay spike gain $G_{s p}^{0}(f)($ Fig $3 \mathrm{C}$ and D). The effective impedance is calculated as the Fourier transform of output voltage divided by the Fourier transform of input current (see Models and Methods). The zero-delay spike gain is the ratio of zero-delay dynamic gain and effective impedance: $G_{s p}^{0}(f)=\frac{G^{0}(f)}{Z_{e f f}(f)}$.

For the type II model, the effective impedance at low frequencies varies substantially with the working point, increasing more than ten-fold at $1 \mathrm{~Hz}(\mathrm{Fig} 3 \mathrm{C}$, upper panel). In a completely passive model without voltage dependent conductance, the impedance does not depend on the input statistics. The observed changes in effec- 
tive impedance, therefore, result from varying degrees of ion channel activity. At the mean-driven working point, the subthreshold voltage fluctuates just below $V_{l o c}$, where some potassium channels are already activated. Depolarization activated potassium current opposes further depolarization, lowering the impedance. This effect is strong for low frequencies, in which the potassium channel activation can follow the input changes, causing the large drop in the orange impedance curve. At higher frequencies, the potassium channels do not actively oppose depolarization. In the limit of frequencies much higher than their activation time constant, the channels present merely a passive leak and their influence on impedance is much smaller, such that the effective impedance decays with the slope of the passive model. The impedance peak around the frequency of the intrinsic firing rate probably results from subthreshold resonance. Increasing $\sigma_{I}$ and lowering $\mu_{I}$ shifts the average voltage to regions where fewer potassium channels are activated. This increases the effective impedance at low frequency (Fig $3 \mathrm{C}$, blue continuous line) and reduces the resonance around $40 \mathrm{~Hz}$. Together, these changes contribute to the $100 \mathrm{~Hz}$ wide bandwidth of $Z_{\text {eff }}$ at the intermediate, fluctuation-driven working point. At the very fluctuation-driven working point, the subthreshold fluctuations are even less shaped by potassium current. The effective impedance in this condition becomes a low-pass filter, determined by the passive neuronal properties (Fig $3 \mathrm{C}$, green continuous line). The type I model, in comparison, has less active ion conductances in the subthreshold range. Therefore, $Z_{\text {eff }}(f)$ is far less sensitive to input conditions. At both fluctuation-driven working points, $Z_{\text {eff }}(f)$ behaves similar to a passive filter with a $10 \mathrm{~Hz}$ cutoff (Fig $3 \mathrm{C}$, blue and green dashed lines).

At first glance, the second subthreshold component, the zero-delay spike gain varies similarly for both models as the working points are changed. It attains larger values close to mean-driven conditions and drops with increasing $\sigma_{I}$ (Fig 3D). This overall behavior is dictated by the condition of constant firing rates. As $\mu_{I}$ decreases and $\sigma_{I}$ increases, larger and larger voltage fluctuations generate the same firing rate, leading to a decrease in zero-delay spike gain. Another general feature of all zero-delay spike gain curves is their monotonic, power law increase in the high frequency limit. It compensates the decay in the effective impedance to reproduce the almost stationary zero-delay dynamic gain (Fig 3D).

Besides these general trends, the shape of the zero-delay spike gain changes between working points, in particular in the low frequency region. For the type II model, a high plateau forms towards the mean-driven condition (orange). Conversely, a sag forms at the extremely fluctuation-driven working point (green). We will see below, that the zero-delay spike gain is the one component, that is most sensitive to the firing pattern. It represents the neuron's propensity to turn voltage fluctuations into firing rate fluctuations based on the intrinsic dynamics. It could therefore be considered surprising, that the zero-delay spike gains of these two working points show opposite shapes. After all, both working points are characterized by bursty firing patterns, evident from the high $C V_{I S I}$ values and the $I S I$ distributions (Fig $2 \mathrm{E}$ ). The difference lies in the source of the bursts. At the mean-driven working point, relatively small amplitude low frequency components drive bursts, fired at the intrinsic frequency. At the very fluctuation-driven working point, large fluctuations of the extrinsic stimulus cause bursts with a intra-burst firing rate that is almost twice as high. The increased stimulus fluctuation size, together with a lower number of APs within bursts causes the zero-delay spike gain to drop at low input frequencies. This will be explored further, when we analyze how repetitively fired, intra-burst APs contribute to the dynamic gain.

The decomposition of $G(f)$ curves across a large range of firing regimes has shown that under most conditions the initiation dynamics does not limit the overall encoding bandwidth, not even in the type I model with slower suprathreshold dynamics. The variable $G(f)$ curves of the type II model and its ability for high bandwidth encoding are explained by a strong stimulus dependence of $Z_{\text {eff }}(f)$ and $G_{s p}^{0}(f)$. Towards large fluctuations, both model types behave very similarly, because the extrinsic stimulus fluctuations dominate the entire signal transformation process, leading to similar $Z_{\mathrm{eff}}(f), G_{s p}^{0}(f)$ and $\frac{G(f)}{G^{0}(f)}$. Decomposing the 

available under aCC-BY-NC-ND 4.0 International license.

subthreshold and suprathreshold impact on dynamic gain, we can also aid the attribution of $G(f)$ changes to a particular parameter change, as we next study the influence of input correlations and neuron morphology. 

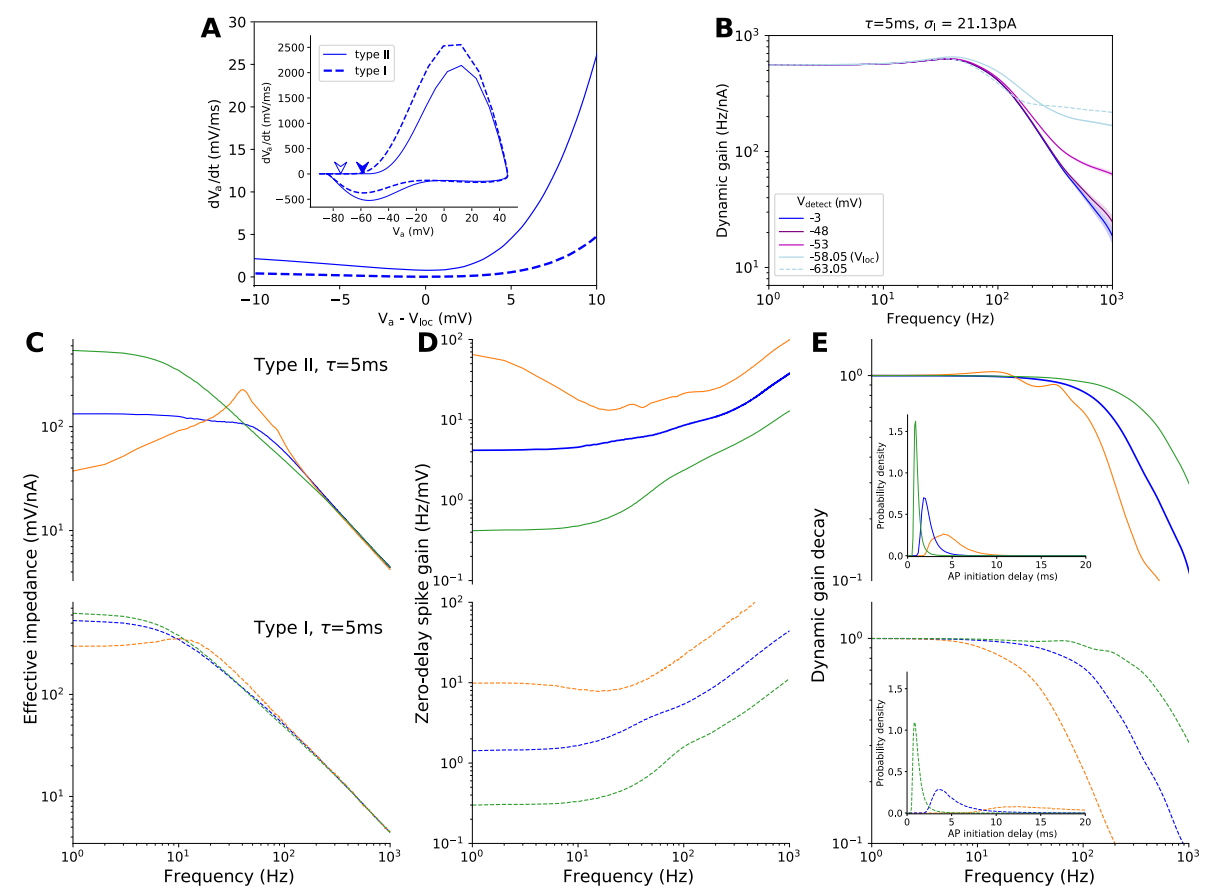

Figure 3: Dynamic gain decomposition based on the decomposition of the AP initiation process. A Insets show the AP phase plots in response to constant inputs just above rheobase. Arrows indicate the positions of the local minima $V_{l o c}$, of type II $(-58.05 \mathrm{mV})$ and type I $(-74.23 \mathrm{mV})$. The region at the beginning of AP initiation is magnified with voltages shifted to align the $V_{l o c}$ values to $0 \mathrm{mV}$. B Reducing the AP detection voltage $V_{\text {detect }}$ towards $V_{l o c}$, enhances the dynamic gain in the high frequency region, exemplified here for the type II model. The zero-delay dynamic gain function (light blue continuous line) behaves similar to that of a LIF-like model. Further reducing the detection threshold reduces the dynamic gain in the low frequency region (light blue dash line). Note that we only include threshold crossings that continue to a full AP. C, D, E The dynamic gain functions from Fig $22 \mathrm{~F}$ and I are decomposed into effective impedance $(\mathrm{C})$, zero-delay spike gain (D) and dynamic gain decay (E). Inset panels in $\mathrm{E}$ are the AP initiation delay distributions at corresponding working points, color code as in Fig 2 Effective impedance captures the transformation from somatic current to axonal voltage $V_{a}$. Zero-delay spike gain is the ratio between zero-delay dynamic gain and effective impedance. Dynamic gain decay is the ratio between original dynamic gain and zero-delay dynamic gain (see Models and Methods). AP initiation delay is the time interval between voltage crossing $V_{\text {detect }}$ (original AP time) and the last previous positive crossing of $V_{l o c}$. 


\section{Fixing the suprathreshold impact reveals the influence of input correlations on dynamic gain.}
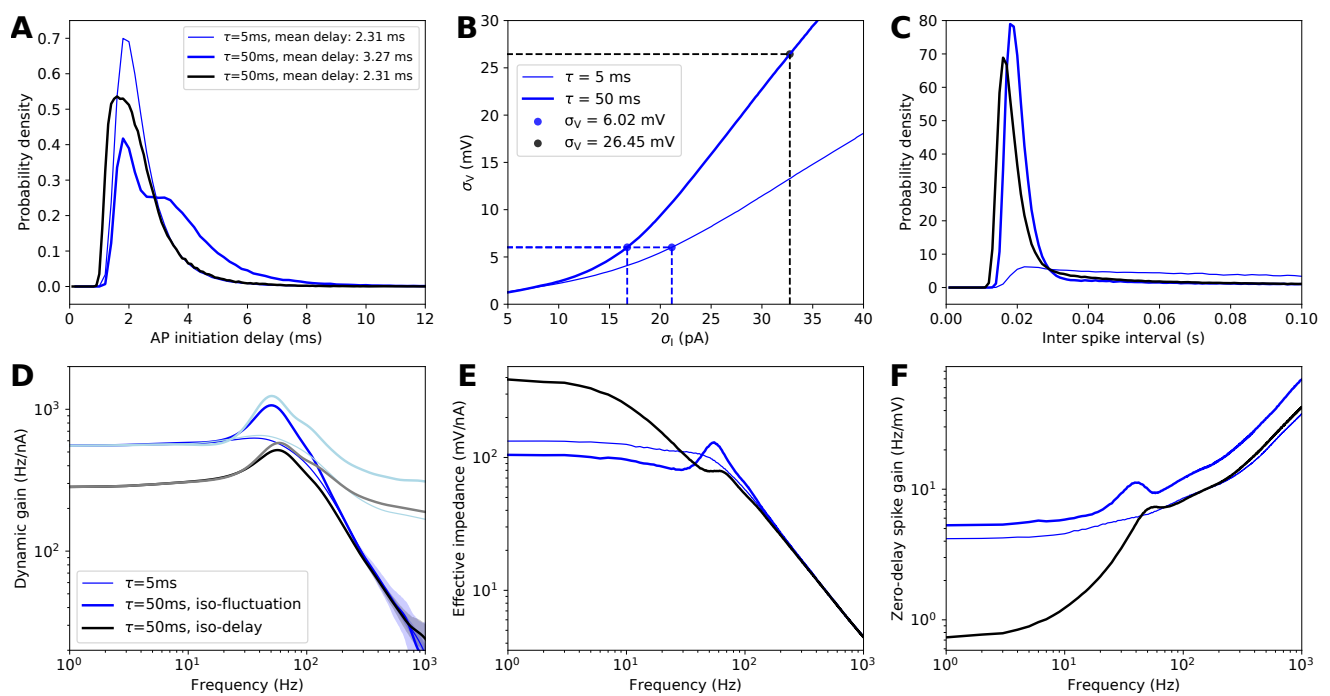

Figure 4: Brunel effect evaluated with two criteria fixing the sub- and suprathreshold impact on dynamic gain. A AP initiation delay distributions of three working points of Eyal's type II model. Two share the same mean delay, denoted as the iso-delay working points (the thin blue curve with $\tau=5 \mathrm{~ms}$ and the thick black curve with $\tau=50 \mathrm{~ms}$ ). The thin and thick blue curves share the same $\sigma_{V}$ indicated in $\mathrm{B}$, denoted as the iso-fluctuation working points. $\mathbf{B} \sigma_{V^{-}} \sigma_{I}$ relations with $\tau=5$ and $50 \mathrm{~ms}$. The scattered data points are the $\sigma_{V}$ of working points in A. The iso-fluctuation working points share the same $\sigma_{V}$ at $6.02 \mathrm{mV}$. $\mathbf{C}$ to $\mathbf{F}$ ISI distributions, dynamic gain and zero-delay dynamic gain functions, effective impedances, zero-delay spike gain functions of the three working points above. Brunel effect, and the constituting sub- and suprathreshold contribution are different when evaluated at the iso-delay and iso-fluctuation working points.

In the previous section, we studied the encoding abilities of Eyal's type I and II models at working points ranging from mean-driven to strongly fluctuation-driven and found that only the type II model could support high bandwidth encoding. Here we continue to examine this type II model, specifically how its encoding depends on stimulus correlation times $(\tau)$. When $\tau$ is increased, cortical neurons, but also LIF models, show enhanced $G(f)$ at high frequencies, as compared to low frequencies $3,9,10,19$. We have called this type of input dependence the 'Brunel effect' and considered it an important feature of real neurons. For our study, we use the dynamic gain function in Fig $2 \mathrm{~F}$ (blue curve, $\tau=5 \mathrm{~ms}$ ) as a reference, and compare it to results obtained with more slowly fluctuating input with $\tau=50 \mathrm{~ms}$.

When we set out to define the exact working points for this juxtaposition, we concluded that $C V_{I S I}$ is of limited practicality, because its non-monotonic $C V_{I S I}-\mu_{I}$ relation in the type II model (Fig $2 \mathrm{E}$ ) does not allow a unique definition of working points. The dynamic gain decomposition motivates two other criteria for working point selection. While a constant $C V_{I S I}$ aims to obtain a comparable output statistics, i.e. a comparable firing pattern, we propose to also study isofluctuation and iso-delay working points. They aim at a comparable subthreshold and suprathreshold dynamics, respectively. The iso-fluctuation working point is found by choosing $\sigma_{I}$ and $\mu_{I}$ to fix $\sigma_{V}$, the standard deviation of subthreshold voltage fluctuations. This is closely related to criteria used in several experimental studies, where $\sigma_{V}$ and the target firing rate have been used to define the input 


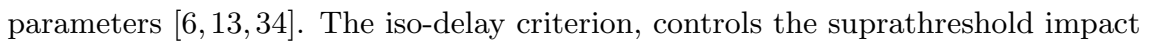
by fixing the average AP initiation delay. Because the intrinsic AP initiation dynamics changes not much between different working points, fixing the average initiation delay for inputs with different $\tau$ values, effectively fixes the impact of the extrinsic currents on AP initiation delay. Fig $4 \mathrm{~A}$ to $\mathrm{C}$ show the AP initiation delay distributions, $\sigma_{V}$, and ISI distributions of the working point with $\tau=5 \mathrm{~ms}$, and its iso-fluctuation and iso-delay counterparts with $\tau=50 \mathrm{~ms}$.

Comparing the dynamic gain functions at the iso-fluctuation working points (Fig $4 \mathrm{P}$, thin and thick blue lines), we find that increasing $\tau$ merely introduces a resonance around $50 \mathrm{~Hz}$, consistent with the ISI distribution peak around 20 millisecond (Fig $4 \mathrm{C}$ ). In the low frequency region, the two gain curves basically coincide, suggesting that the iso-fluctuation criterion indeed equalizes the subthreshold dynamics across the two input conditions. Because the dynamic gain curves in the high frequency region are also nearly identical, one could conclude that the Brunel effect is absent under iso-fluctuation conditions. However, a comparison of the zero-delay dynamic gain functions (Fig $4 \mathrm{D}$, wide and thin light blue curves), does show improved high frequency encoding, for $\tau=50 \mathrm{~ms}$ as compared to $\tau=$ $5 \mathrm{~ms}$. This means that the dynamic gain decay for $\tau=50 \mathrm{~ms}$ is larger, which is consistent with the more variable AP initiation delay (Fig $4 \mathrm{~A}$ ).

Next, we examined the Brunel effect at the iso-delay working points. With the same average AP initiation delay, the overall shapes of the AP initiation distributions are also similar (Fig 4 A thin blue line and grey line). Achieving similar initiation delays with much longer input correlations requires a larger $\sigma_{I}$. $\sigma_{V}$ is even more than quadrupled from 6 to $26 \mathrm{mV}$ (Fig $4 \mathrm{~B}$ ). But even the drastic increase in voltage fluctuations only shifts the ISI distribution slightly to smaller values (Fig $4 \mathrm{C}$ thick lines). The large increase in $\sigma_{I}$ for $\tau=50 \mathrm{~ms}$ results in a strong reduction of the dynamic gain function in the low frequency region (Fig $4 \mathrm{D})$. It now lies below the dynamic gain function for $\tau=5 \mathrm{~ms}$. The high frequency limits of the two $G(f)$ curves are close to each other, and even the zero-delay gain functions $G^{0}(f)$, now attain similar values in the high frequency limit. We conclude that the type II model reproduces the Brunel effect at the iso-delay working points, which fix the impact of suprathreshold dynamics on population encoding. In this condition, the relative improvement of high frequency encoding observed in the normalized dynamic gain functions is realized by reducing the unnormalized dynamic gain in the low frequency region when $\tau$ is larger. At iso-fluctuation working points, on the other hand, the low frequency encoding is nearly constant and no Brunel effect manifests in the dynamic gain curves because the stronger dynamic gain decay cancels increased zero-delay dynamic gains at $\tau=50 \mathrm{~ms}$.

We can now use the dynamic gain decomposition to check whether iso-fluctuation and iso-delay criteria indeed standardize sub- and suprathreshold contributions, and to further understand the source of the Brunel effect (Fig $4 \mathrm{E}$ and F). We find that the effective impedances and zero-delay spike gains are very similar at the iso-fluctuation working points, in accordance with its purpose of fixing subthreshold dynamics. Within the identical subthreshold voltage fluctuation range, the active ion conductances recruited for stimulus filtering are close to each other. The remaining difference probably stems from the slightly more depolarized average voltage for $\tau=50 \mathrm{~ms}$, leading to slightly higher potassium current activation. Two zero-delay spike gain curves are almost paralleled with each other, indicating that the voltage frequency components are transformed into firing frequency components in similar ways for both correlation times. However, at the iso-delay working points, the effective impedance and spike gain in the low frequency region show large but opposite changes in response to increasing $\tau$. While the effective impedance at $1 \mathrm{~Hz}$ is more than 3 times larger for $\tau=50 \mathrm{~ms}$, the spike gain at $1 \mathrm{~Hz}$ is more than 5 times larger for $\tau=5 \mathrm{~ms}$. The difference in effective impedance is readily explained by the difference in average potassium channel conductance. $\sigma_{V}$ is about $6 \mathrm{mV}$ for $\tau=5 \mathrm{~ms}$, and increases to about $26 \mathrm{mV}$ for $\tau=50 \mathrm{~ms}$. The substantially larger fluctuations into the hyperpolarized range cause ion channel deactivation, a lower membrane conductance and thereby a larger effective impedance. This is reflected 
in the much lower cutoff frequency of $8.3 \mathrm{~Hz}$ for $\tau=50 \mathrm{~ms}$ as compared to the $56 \mathrm{~Hz}$ for $\tau=5 \mathrm{~ms}$. Low-pass effective impedance keeps more low frequency components of stimulus in the voltage fluctuations. As a result, the zero-delay spike gain at the iso-delay working point is drastically reduced in the low frequency region (Fig $4 \mathrm{~F}$, black curve). The decomposition of zero-delay dynamic gain functions implies two different ways to realize high bandwidth encoding for two correlation times of input, by influencing the firing patterns emitted by the neuron. How that impacts the dynamic gain will become clear, when we next study the differential encoding capacity of fluctuation-driven APs and repetitively firing APs.

\section{Decomposing dynamic gain functions into repetitive fir- ing and individual AP components.}

Our previous arguments suggest that the firing pattern, in itself, is an important contributor to the shape of the dynamic gain function. It could even be thought that the type II model is capable of high bandwidth encoding in part due to its ability to fire high frequency repetitive APs. We next attempt to analyze this more stringently, by decomposing the dynamic gain function into two parts, contributed by two AP populations (Fig 5 A).

One population is fluctuation-driven APs, defined as those fired after at least $50 \mathrm{~ms}$ of silence. These APs are likely to be the first one, or the only one, fired in a voltage upstroke, while the rest of APs are likely part of a burst later in an upstroke, denoted as repetitive firing APs. Fig 5A is an illustration of the spike classification. The relative fraction of the two components differs between correlation times, as indicated by the firing rates in the figure legend. When $\tau=50 \mathrm{~ms}$, the ISI distribution is significantly more peaked around $25 \mathrm{~ms}$ (see Fig $4 \mathrm{C}$ ), and more than half of the APs are classified as repetitively firing APs. This fraction drops to $20 \%$ for $\tau=5 \mathrm{~ms}$, because bursts are terminated by the rapid $V_{a}$ fluctuations.

We now calculated dynamic gain functions based on APs from only fluctuationdriven or only repetitive firing APs. When their phases are taken into account, these two dynamic gains can be summed to yield the curves in Fig $4 \mathrm{P}$ (black and thin blue). The fluctuation-driven APs alone encode the input with a higher bandwidth, as compared to all APs together (Fig $5 \mathrm{~B}$ vs iso-delay working points in Fig 4D). The corresponding cutoff frequencies are $133 \mathrm{~Hz}$ vs $105 \mathrm{~Hz}$ for $\tau=$ $5 \mathrm{~ms}, 234 \mathrm{~Hz}$ vs $166 \mathrm{~Hz}$ for $\tau=50 \mathrm{~ms}$. The shapes of these gain curves reflect the broad 20 to $50 \mathrm{~Hz}$ frequency encoding preference of the fluctuation-driven APs. When the intrinsic preference is overridden by very strong external fluctuations, this preference is suppressed (see supplementary Fig S4).

The repetitive firing APs alone lead to dynamic gain curves with very pronounced resonance around the preferred firing rate and a pronounced dip around half of that frequency (Fig $5 \mathrm{C}$ ). The dip is a direct consequence of the resonance, because APs locked to an input component at the resonance frequency and fired in a short burst will be locked worse than random to half of that frequency. The dynamic gain functions derived from repetitive APs alone have a comparatively narrow bandwidth, especially for $\tau=5 \mathrm{~ms}$, where the cutoff frequency is below $8 \mathrm{~Hz}$. In conclusion, the two dynamic gain functions for $\tau=5$ and $50 \mathrm{~ms}$ at the iso-delay working points realize high bandwidth encoding in different ways. One reduces the fraction of repetitive firing APs, while the other forms longer bursts of repetitive firing APs to weaken its low-pass filter effect. In either case, our results clearly show that the high encoding bandwidth of Eyal's model with type II excitability is not a direct result of the burst firing. APs fired within only contribute the pronounced peaks at the preferred firing rate. We can also use the AP classification strategy to further investigate the variation of spike gain shape across working points. Low frequencies were particularly represented in the zero-delay dynamic gain curve, when the neuron was nearly mean-driven (Fig 3D, orange). As supplementary Fig $\mathrm{S} 4$ shows, this is caused by a relatively large fraction of repetitive firing APs. They 

available under aCC-BY-NC-ND 4.0 International license.

occur in long bursts and thereby limit the encoding of intermediate frequencies. This can be understood when considering the example of a $100 \mathrm{~ms}$ long burst, for which first and last APs fall on diametral phases of a $5 \mathrm{~Hz}$ stimulus component and thereby limit encoding of this frequency. In contrast, the fluctuation-driven APs, do not show such a preference for very low frequencies. Next, we will use the dynamic gain decomposition method to examine the impact of dendrite size on population encoding. 

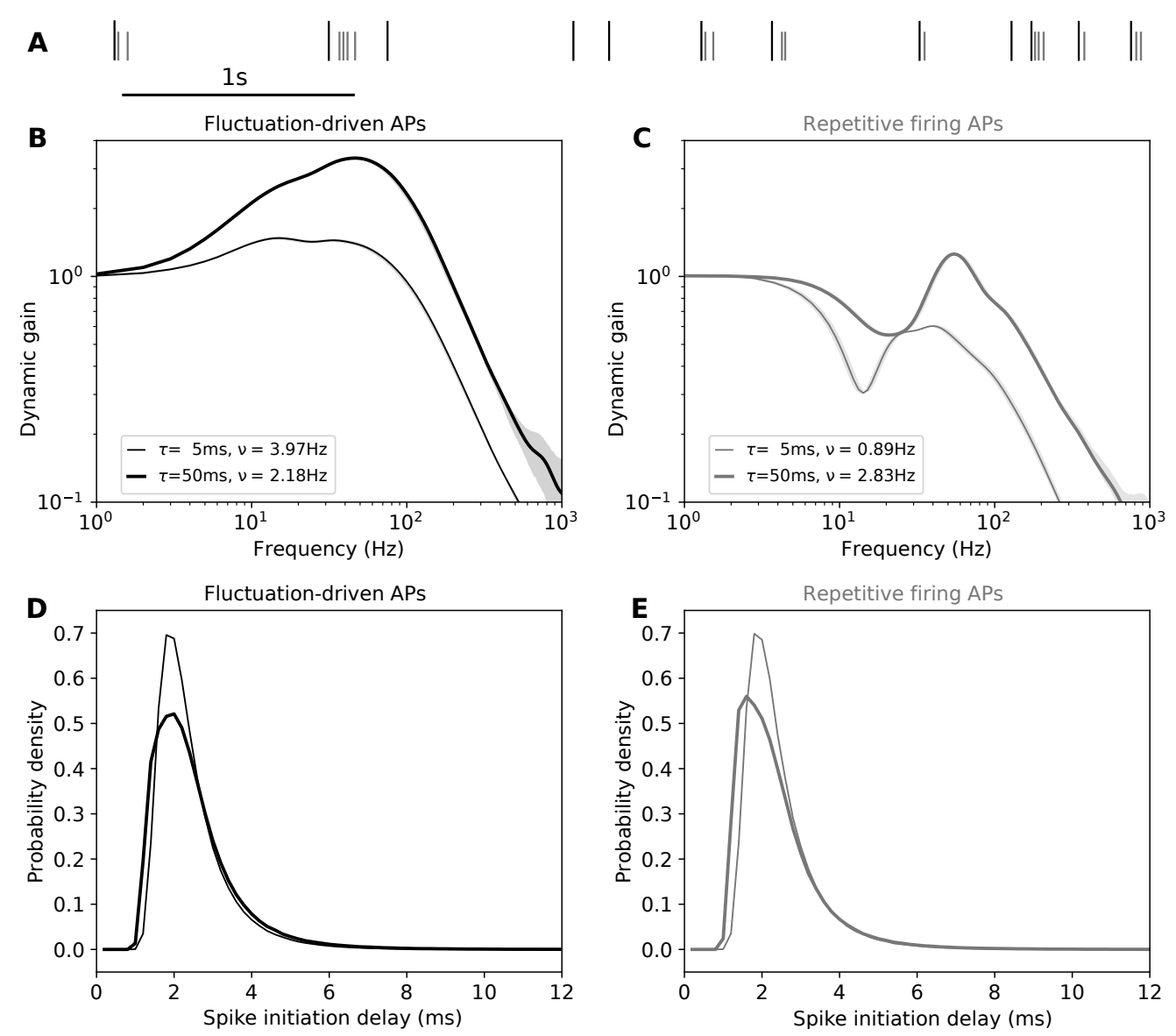

Figure 5: Intrinsic high-frequency firing undermines high bandwidth encoding. A A short sequence of APs from the simulations in Fig 4 ( $\tau=50 \mathrm{~ms}$, iso-delay), shown in a raster plot. The APs are classified into two groups according to the preceding ISI (ISI $\geq 50 \mathrm{~ms}$ black, longer dashes, ISI $<50 \mathrm{~ms}$ grey shorter dashes). Separate dynamic gain functions of the two iso-delay dynamic gain functions in Fig 4 are calculated for these two spike classes. B The dynamic gain curves derived from fluctuation-driven APs. High frequency input components are encoded better than low frequency components. C The dynamic gain curves derived from APs fired in close succession, e.g. repetitively fired APs. Resonance effects cause the dips and peaks (see main text). D and $\mathbf{E}$ The AP initiation delay distributions of the two spike classes are very similar, and hence very similar to the joint distribution in Fig 4 A. See supplementary Fig S3 for the corresponding analysis at the iso-fluctuation working points. 


\section{Dendrites enhance high bandwidth encoding by suppress- ing low frequency effective impedance.}
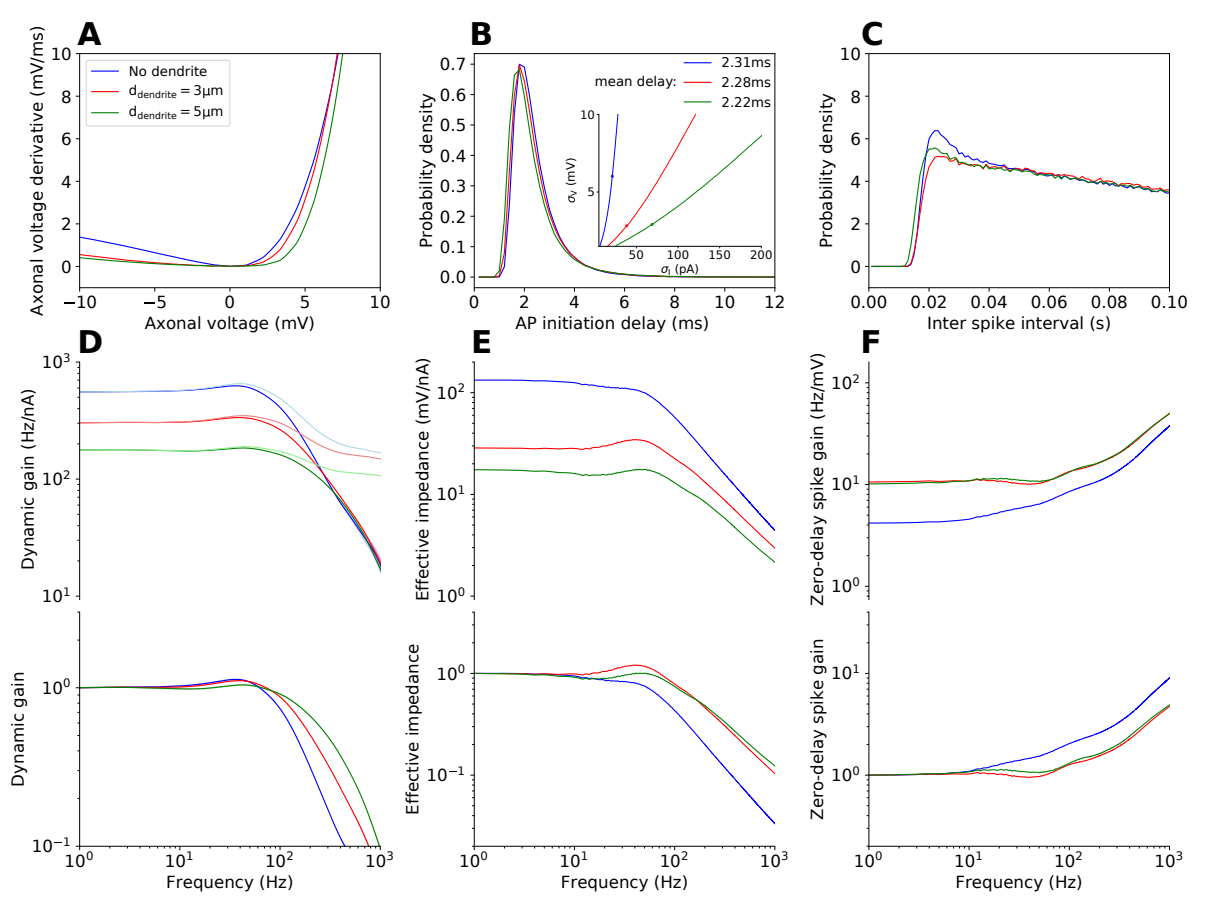

Figure 6: Fixing the AP initiation delay, a larger dendrite can enhance high frequency encoding through effective impedance. The spike gain undermines this enhancement. A Phase plots of three model variants with different dendrite sizes, $V_{l o c}$ aligned at $(0 \mathrm{mV}, 0 \mathrm{mV} / \mathrm{ms})$. B AP initiation delay distributions at the iso-delay working points $(\nu=5 \mathrm{~Hz})$. Inset panel shows corresponding $\sigma_{V}$ on $\sigma_{V^{-}} \sigma_{I}$ relations. C ISI distributions at the iso-delay working points. D, E, F Dynamic gain functions and zero-delay dynamic gain functions (D), effective impedances (E) and zero-delay spike gain $(\mathrm{F})$ of the three model variants calculated at iso-delay working points (upper panels), together with their normalized gain curves (lower panels). See supplementary Fig S6 for the results with $\tau=50 \mathrm{~ms}$.

To understand the impact of dendrites on population encoding, we took the two model variants with a median and a large dendrite from [1] for comparison (see Models and Methods), both display type II excitability (see supplementary Fig S5). We first examined the impact of dendrite size on intrinsic AP initiation dynamics. Increasing the dendrite size increases the lateral current from the initiation site towards the soma, due to the larger somato-dendritic current sink. $V_{l o c}$ is shifted to slightly more depolarized values from $-58.05 \mathrm{mV}$ to $-54.72 \mathrm{mV}$ and $-53.61 \mathrm{mV}$. As a result, the AP onset is shifted towards voltages at which the sodium channels have steeper voltage dependence. Consequently, at later stages, e.g. $10 \mathrm{mV} / \mathrm{ms}$, the slope of the phase plot is actually larger for models with larger dendrites, as has been previously reported in [11] (see Fig 6A). However, when aligning $V_{l o c}$ to $(0 \mathrm{mV}, 0 \mathrm{mV} / \mathrm{ms})$, we found that a larger dendrite does not accelerate AP initiation close to $V_{l o c}$, instead, the local slope of the phase plot is reduced. A first evaluation of the subthreshold effects of the dendrites can be gained from the $\sigma_{V}$ $\sigma_{I}$ relation (Fig $6 \mathrm{~B}$, inset panel). The large current sink of the dendrite drastically decreases the impedance of the neuron, reflected by the reduced slopes. Compared to this drastic impedance effect, the dendrite's impact on the suprathreshold dynamics appears to be subtle. Therefore, to compare the population encoding 
capabilities of the three models, we chose iso-delay working points to harmonize the suprathreshold contribution and focus on different subthreshold contribution of the dendrite.

Taking the dynamic gain function of $\tau=5 \mathrm{~ms}$ in Fig 4 for reference, we fixed the average AP initiation delay for the three model variants, leading to very similar distribution shapes (Fig 6B). Under this criterion, $\sigma_{V}$ is smaller for the neuron model with a dendrite (scattered dots in inset panel), and the three ISI distributions are similar to each other (Fig 6C). Compared to the dynamic gain function without the dendrite (blue), the other two gain curves (red and green) have smaller dynamic gain below $200 \mathrm{~Hz}$. In the high frequency region, gain curves decay with similar trends, and corresponding zero-delay dynamic gain functions are also close to each other (Fig 6D, upper panel). Normalizing the dynamic gain functions, we observed an enhancement of high frequency encoding with a larger dendrite (lower panel in D).

Decomposing the zero-delay dynamic gain functions into effective impedances and zero-delay spike gains, we found that adding a dendrite does not reduce the effective impedance equally across all frequencies (Fig 6 E, upper panel). Instead, low frequency components are suppressed more strongly, because they charge a larger portion of the dendrite, while high frequencies experience much stronger spatial filtering and charge only the proximal dendrite. When the effective impedances are normalized (lower panel in E), the dendrites suppression of low frequencies appears as a boost of high frequency representation in the voltage. Interestingly, a further substantial increase in the dendrite size hardly affects the shape of the effective impedance. The zero-delay spike gain curves are relatively flat in the low frequency region, and they increase in similar trends in the high frequency region (Fig 6F, upper panel). The curves for the two dendrite-bearing models are almost identical (red and green), while the zero-delay gain curve of the original model has lower values (blue), because it produces very similar firing patterns (Fig 6C) with approximately three times larger subthreshold voltage fluctuations (inset in Fig 6B). The normalized curves in the lower panel show that, the dynamic gain enhancement caused by the effective impedance is slightly undermined at the stage of zero-delay spike gain (from blue to red, and green). Taken together, the improved high frequency encoding in the presence of a dendrite is primarily due to a suppression of low frequencies in the effective impedance, with a minor effect of the accelerated AP initiation, leading to weaker dynamic gain decay. Studying the effect of the dendrite under slower input correlations, led to the same conclusion. In particular, the dendrite did not change the Brunel effect (supplementary Fig S6).

\section{Subcellular dissection of a pathophysiological assault on population coding by dynamic gain decomposition.}

The dynamic gain decomposition is designed to be applicable to any neuron, independent of its complexity. It should be applicable even to recordings from real neurons, provided that the recorded somatic voltage contains sufficient information. For the subthreshold analysis, the calculation of the effective impedance, this seems likely. The utility of somatic recordings for initiation delay is not clear a priori. We therefore set out to apply the dynamic gain decomposition to experimental data and chose a specific data set from a previous study for two reasons. First, the two groups in the data set had been reported to have different input resistances, prompting us to expect different effective impedances. Second, the treatment group contained neurons with very different dynamic gain curves, which we would like to understand better by decomposition.

The data in question were recorded from layer 5 pyramidal neurons in coronal slices of mouse somatosensory cortex and originally published in 5]. Slices in the treatment group underwent two brief hypoxic episodes that induced spreading depolarization. Controls did not experience hypoxia. Unlike simulations, experi- 
ments record voltage at the soma and not the initiation site. We showed earlier, that the transfer impedance from soma to initiation site does pose an additional filter for higher input frequencies. However, for distances up to $50 \mu \mathrm{m}$, this effect was very small [26], and hence we speculate that using the somatic voltage will still allow decomposition.

We decomposed the dynamic gain functions of each pyramidal neuron into effective impedance and spike gain. For two, very differently affected neurons from the treatment group of [5], the results are shown in Fig 7 Neuron 1 displays encoding with a bandwidth of approximately $350 \mathrm{~Hz}$ (Fig 7A). Its dynamic gain's confidence interval widens substantially, as the gain curve drops below the noise floor. In comparison, the dynamic gain of neuron 2 (Fig $7 \mathrm{~B}$ ) is substantially larger in the low frequency region, decays more steeply at intermediate frequencies, and displays a lower cutoff frequency of around $200 \mathrm{~Hz}$. But surprisingly, above $400 \mathrm{~Hz}$, its dynamic gain reemerges above the noise floor and increases with a smaller bootstrapping confidence interval as compared to neuron 1. Also note that the absolute magnitude of the dynamic gain, for instance at $200 \mathrm{~Hz}$, is higher in neuron 2, although its cutoff frequency is lower. The underlying properties that cause these differences became clear, when we decomposed the dynamic gain into the sub- and suprathreshold components.

As a first step, we estimated the phase plot from the recordings (see Models and Methods) by averaging voltage derivatives at different voltages (inset panels in A and B). This reveals a difference in threshold voltage, but most importantly, a large difference in the amount of voltage sensitive depolarizing currents between the two neurons. For neuron 1, the voltage derivative changed little for voltages below $-40 \mathrm{mV}$, then suddenly increased to $30 \mathrm{mV} / \mathrm{ms}$ within $8 \mathrm{mV}$, reflecting large AP initiation currents. Neuron 2, in contrast, had smaller depolarizing currents and a higher threshold. The position of the local minimum $V_{l o c}$ was around $-30 \mathrm{mV}$ for neuron 2 and, although it could not be discerned precisely, it was likely just below $-40 \mathrm{mV}$ for neuron 1 .

In the second step, we reduced the AP initiation delay by lowering the AP detection threshold $V_{\text {detect }}$ analogous to the analysis of Eyal's model in Fig $3 \mathrm{~B}$. The recalculated dynamic gain functions for the various $V_{\text {detect }}$ values are given in the lower panels of Fig $7 \mathrm{~A}$ and B. For neuron 1, the gain curves changed abruptly, once $V_{\text {detect }}$ reached $-40 \mathrm{mV}$ and already at $-45 \mathrm{mV}$ a saturation was reached. Apparently, only this narrow supra-threshold voltage range contributed to the uncertainty in AP timing. This corresponds to the steep change in the phase plot (inset). The delay variability caused a decay in the dynamic gain only for frequency components above $300-350 \mathrm{~Hz}$. When the AP was detected around $V_{l o c}$, the dynamic gain curve resembled that of a LIF neuron model without voltage dependent initiation and with a hard threshold [19. This result replicates the simulations shown in Fig $3 \mathrm{~B}$, albeit with an almost four times higher bandwidth of the dynamic gain decay, as compared to Eyal's type II model.

In neuron 2, the recalculated dynamic gain curves behaved differently. The transition to LIF-like behavior was much more gradual (Fig $7 \mathrm{~B}$, colored traces). The uncertainty in AP timing accumulated during the transition through a much larger voltage range from above $-25 \mathrm{mV}$ down to $-40 \mathrm{mV}$, mirroring the more gradual change in phase plot slope (inset). Another important difference is the affected frequency range. In neuron 2 , suprathreshold delay variability already reduced the encoding for frequencies above $90-100 \mathrm{~Hz}$, similar to what we reported for Eyal's model.

Our insights into the different suprathreshold dynamics are complemented with an analysis of the subthreshold contribution to dynamic gain. We calculated the effective impedance as the ratio of Fourier transform of voltage and Fourier transform of current (see Models and Methods). The two neurons' effective impedances and zero-delay spike gains are juxtaposed in Fig 7 $\mathrm{C}$ and D. This reveals the considerably larger effective impedance of neuron 2 as the origin of this neuron's large absolute dynamic gain value. The fact that this effective impedance rises for frequencies above $30 \mathrm{~Hz}$ is puzzling and we can only speculate about 
a contribution of sodium channels, possibly due to the increased threshold in neuron 2. For neuron 1, the effective impedance approximates the shape one would expect from a passive soma with dendrites. It is dominated by a cutoff of $12.8 \mathrm{~Hz}$, i.e. a membrane time constant of $12.5 \mathrm{~ms}$. Beyond the cutoff, the slope is less negative than -1 , most likely due to the presence of dendrites. The zero-delay spike gains of the two neurons are rather similar at low frequencies, but above $20 \mathrm{~Hz}$, they deviate increasingly. Because these curves are obtained by dividing zero-delay dynamic gain curves by the effective impedance, this deviation originates in the unusual impedance increase in neuron 2.

In summary, the differences between the two neurons' dynamic gain curves can be attributed to subthreshold and suprathreshold contributions as follows: the difference in absolute values is caused by the higher effective impedance of neuron 2. The different locations of the dynamic gain drop, and consequently the different bandwidths are explained by the substantially slower AP initiation in neuron 2. It causes an earlier drop in dynamic gain due to larger initiation delay variability. The remaining difference is the striking rise in the high frequency region of the second neuron's dynamic gain. We suspect that at the high frequencies the extrinsic, stimulation-induced currents dominate the second neuron's weaker intrinsic currents. Consequently, the exact time of threshold crossing is influenced by high frequency input fluctuations, similar to the situation in a LIF neuron model. This is the reason for the rising dynamic gain and the relatively narrow confidence interval. In the context of the original study [5], it is interesting to note, that cells with such a weak intrinsic AP initiation appeared only after hypoxia and spreading depolarization, which also compromise the molecular integrity of the axon initial segment.

Applying dynamic gain decomposition to all the cells in the original data set, we could further investigate the previously described effect of hypoxia-induced spreading depolarization on the dynamic gain. The grand averages for dynamic gain $\langle G(f)\rangle$, effective impedance $\left\langle Z_{e f f}(f)\right\rangle$ and spike gain $\left\langle G_{s p}(f)\right\rangle$ were calculated over the frequency range, where data where available from all cells. For $\langle G(f)\rangle$ and $\left\langle G_{s p}(f)\right\rangle$ that only includes the range up to $300 \mathrm{~Hz}$, at which point at least one cell's $G(f)$ touched the noise floor. The grand averages, together with their standard errors are shown in Fig $7 \mathrm{E}$. They show clearly that the average effective impedance is increased in the treated cells, and that this difference dominates the unnormalized dynamic gain curves. While the dynamic gain of the treated cells appears to drop earlier, between 100 and $200 \mathrm{~Hz}$ as compared to the control cells $>300 \mathrm{~Hz}$, this difference does not manifest in a statistically significant difference. The dynamic gain values at $200 \mathrm{~Hz}$ are spread out over similar ranges for both groups (Fig 7F). In contrast, the impedance-corrected spike gain values are significantly different (Student's t-test, $\mathrm{p}=0.048$ ). These examples show that all aspects of dynamic gain decomposition can be applied to experimental data and serve to suggest biophysical parameters as the basis of individual dynamic gain features. 

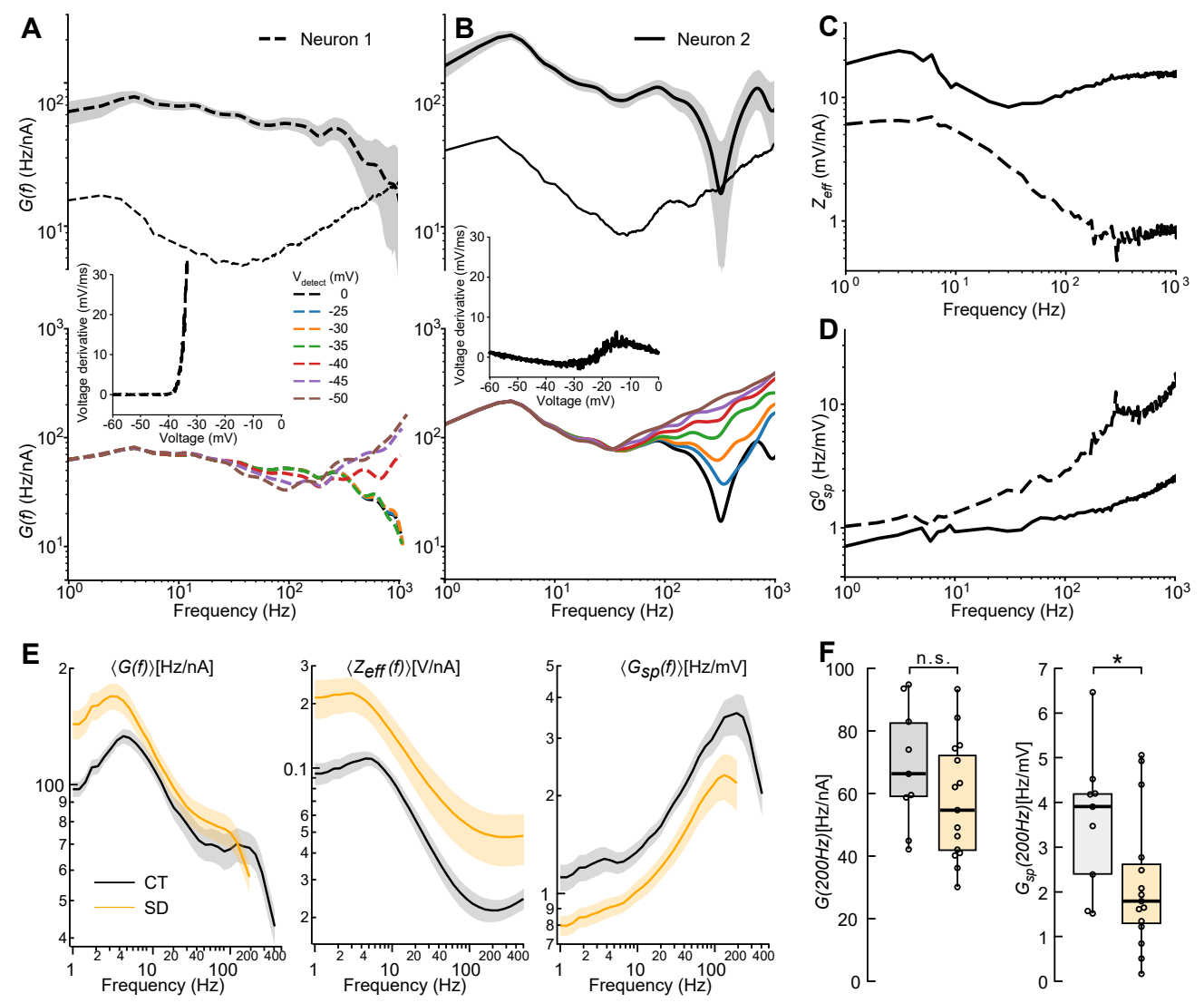

Figure 7: An interpretation on different encoding abilities in cortical neurons with dynamic gain decomposition. A Dynamic gain function $G(f)$ (upper panel) of one pyramidal neuron from the treatment group in [5], and the associated gain curves recalculated with lower $\mathrm{AP}$ detection thresholds ranging from $0 \mathrm{mV}$ to $-50 \mathrm{mV}$ (lower panel). Inset panels are the phase plots estimated by averaging voltage derivatives at each voltage value. Note the wide bandwidth of the original gain function. Grey areas are the 95 th percentile bootstrapping confidence intervals. Thin line represents the 95 th percentile noise floor (see Models and Methods). B as A, but for another neuron from the treatment group with drastically different gain. Firing rates are $4.10 \mathrm{~Hz}$ and $4.68 \mathrm{~Hz}$ in $\mathbf{A}$ and $\mathrm{B}$ respectively. $\mathbf{C}$ and $\mathbf{D}$ Effective impedances and zero-delay spike gains of the dynamic gain functions in A and B. $G_{s p}^{0}$ is the ratio between zero delay dynamic gain ( $V_{\text {detect }}=-50 \mathrm{mV}$ in $\mathrm{C}$ and $\left.\mathrm{D}\right)$ and effective impedance. E Grand average of $G(f)$, $Z_{\text {eff }}$, and $G_{s p}(f)$ for control neurons $(\mathrm{CT}, \mathrm{n}=9)$ and neurons undergoing brief hypoxia and spreading depolarization $(\mathrm{SD}, \mathrm{n}=15)$. Lines and shades represent means and their standard error. $G(f)$ and $G_{s p}(f)$ are displayed for the frequency region in which all individual traces are significant, i.e. above the respective noise floor. $\mathbf{F} G(f)$ at $200 \mathrm{~Hz}$ does not differ between groups. Due to the large $Z_{\text {eff }}$ differences between the groups, the spike gains at $200 \mathrm{~Hz}$ are significantly different (t-test, ${ }^{*} \mathrm{p}=0.048$ ). 


\section{Discussion}

In this work, we developed a straightforward method to decomposed the contribution of subthreshold and suprathreshold dynamics on population encoding. It allows for a unified and comprehensive approach of dynamic gain analysis because it can be applied to simulated data from models of any complexity and even measurements from real neurons. When we applied the dynamic gain decomposition to a complex multi-compartment model equipped with biophysically plausible AP initiation mechanisms. We found that the model's high encoding bandwidth is mainly enabled by a high potassium conductance around threshold. This also causes a type II excitability, but the tendency to high frequency bursting is not in itself beneficial for a high bandwidth. The decomposition also guides the definition and choice of working points at which model variants or cell types can be compared without bias. Iso-(voltage-)fluctuation and iso-(initiation-)delay working points control for sub- or suprathreshold contributions, respectively. We found the iso-delay criterion particularly useful for understanding how changes in input correlation and dendrite size affect encoding bandwidth. We also applied the decomposition to recordings from two layer 5 pyramidal neurons, which were differently affected by hypoxia. Their different dynamic gain shapes and bandwidths are resulted from striking differences in effective impedance and intrinsic initiation currents. This demonstrates that dynamic gain decomposition can connect cellular physiology to network function.

Fixing working points isolates parameter effects. The dynamic gain function is not a fixed curve but depends on the working point of the neuron model. It can change substantially when the operating regime is changed from mean-driven to fluctuation-driven (Fig $2 \mathrm{~F}$ ). Thus it is essential to properly align the working points of model variants, such that the differences detected in the dynamic gain functions originate mainly from the targeted parameter change, rather than unrelated working point factors. One criterion we adopted here is fixing the firing rate at $5 \mathrm{~Hz}$. A second criterion can be chosen to fully determine the working point on the two dimensional manifold of firing rate, defined by $\mu_{I}$ and $\sigma_{I}($ Fig 2 C). Previous studies have focused on fixing the subthreshold dynamics, either by fixing the voltage fluctuations $\sigma_{V}$ 3 6, 13, or by fixing the related firing

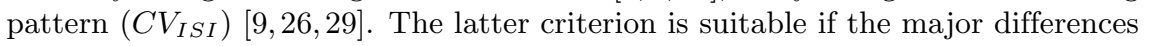
manifest mostly in the suprathreshold regime.

Here, we introduced a complementary criterion to fix the impact of suprathreshold dynamics. We quantified the variability in AP initiation with the initiation delay. By fixing the mean of this random variable, we managed to control the impact of the suprathreshold AP dynamics across models, which corresponds to the dynamic gain decay in the high frequency region. We found this criterion particularly useful for studying the Brunel effect and the dendritic effect on Eyal's model, since both mainly affect the subthreshold dynamics. What is more, this criterion could even be generalized to LIF-like models, in which $V_{\text {detect }}$ is equal to $V_{l o c}$. We propose that our approach of fixing the average AP initiation delay in models with active initiation is equivalent to fixing the average voltage derivative at threshold in LIF-like models. Therefore, the decomposition-inspired working points can be used for all types of models, including those with a threshold and reset.

Dynamic gain function decomposition The decomposition of dynamic gain functions disentangles the complex influence of the model and working points on the shapes of gain curves, which benefits all dynamic gain analyses. To this end, we introduced three components that combine to the dynamic gain: 1 . the effective impedance, 2. the zero-delay spike gain, and 3. the dynamic gain decay due to the stochastic AP initiation delay. The effective impedance captures the passive model properties as well as the average subthreshold activation of ion channels. This sensitivity to ion channel activation reveals a substantial contribution to 
the Brunel effect, the different transformation of low frequency input currents into voltage fluctuations for different correlation times. The zero-delay spike gain describes the transformation from voltage fluctuations to AP times detected at $V_{l o c}$, at a point where the intrinsic AP initiation currents still play a minor role in the voltage dynamics. The zero-delay spike gain therefore captures the influence of the overall firing pattern but excludes the variable AP initiation durations. When the working point is changed in a way that reduces the effective impedance at low frequencies, often the zero-delay spike gain shows a compensatory increase. These opposing effects remain undetected without decomposition. Effective impedance and zero-delay spike gain together capture the impact of subthreshold dynamics on population encoding, and reproduce a LIF-like dynamic gain function. The third component, the dynamic gain decay in the high frequency region, reflects how intrinsic currents influence the AP initiation dynamics, such that the resulting AP timing is less determined by the stochastic input. Within a given neuron model, it explains why the zero-delay gain curves at distinct working points are collapsed to similar decay trends in the high frequency limit. Between different model variants, this understanding also inspires the idea of fixing the mean AP initiation delay to control the impact of suprathreshold dynamics on population encoding.

Our methodology of aligning the working points and decomposing the dynamic gain functions provides a general tool for evaluating various hypotheses on population encoding. The decomposition approach allows identification of sub- and suprathreshold effects, even if the models to be compared are so different that it is not feasible to align the working points.

Encoding abilities of type II neurons The model introduced by Eyal et al. 11] has a relatively wide bandwidth $(>100 \mathrm{~Hz})$ even without dendrites, provided it operates at a fluctuation-driven working point with intermediate input fluctuations (blue trace in Fig 2F). A closely related model with type I excitability displayed a substantially lower bandwidth (approximately 10-15 Hz) throughout the large parameter space we explored. Our dynamic gain analysis identified the mechanisms supporting this high bandwidth encoding. The main effect originates in a larger fraction of potassium channels activated at subthreshold voltages, which reduces the membrane time constant and thereby increases the bandwidth of the effective impedance, as compared to the type I model. This suppression of low-frequency voltage components creates an apparent high-frequency boost. An additional improvement of the high-frequency encoding stems from a higher voltage sensitivity of the AP initiation currents. Although the voltage sensitivity of the sodium and potassium channels does not differ between the type I and type II model, the relative shift in their half-activation voltages means that at threshold, $\frac{d}{d V} \frac{d V}{d t}$ is larger in the type II. Accordingly, the dynamic gain decay's bandwidth is increased and does not limit the overall dynamic gain bandwidth at all tested working points. The typical firing pattern of type II models, high frequency repetitive firing, is of minor importance for the high bandwidth. A stratification of APs according to the firing pattern (isolated or intra-burst), reveals the limited encoding capacity of APs fired during repetitive firing episodes (Fig 5 C). The type II excitability, the low membrane time constant and the bandwidth limitation by impedance but not initiation dynamics, all mark Eyal's model as distinct from layer 5 pyramidal cells. A dynamic gain decomposition of fast spiking interneurons, studied in 10], might show more resemblance to the encoding properties of these neurons.

Experimental studies from several labs have reported that cortical pyramidal neurons are capable of ultrafast, high-bandwidth encoding $3,6,6,12,14,16$. Interestingly, those neurons are clearly of excitability type I. The few experimental studies of type II neurons, namely purkinje cells [15] and fast spiking interneurons 10. have shown that their encoding bandwidth is comparable or even higher that that of pyramidal neurons. 10] also reported the dynamic gain functions of firing rate-adapting interneurons and found a surprisingly strong dependence on the input correlations. The dynamic gain decomposition presented here might be 
instrumental in understanding this flexibility of dynamic gain.

Implications for neuronal network modelling The simulation of multicompartment models is computationally costly and almost prohibitive for large network simulations. However, the shape and bandwidth of the dynamic gain play important roles for studies of information processing in large, recurrent neuronal networks. Our dynamic gain decomposition suggests routes to create simpler models with dynamic gain curves that depend on input statistics just as the full multi-compartment models do. If fixed thresholds and resets are used, the realistic limited bandwidth could be obtained by adding a random AP initiation delay distribution. The subthreshold influence on the dynamic gain curve could be created by a few voltage-gated or AP time dependent conductances that create adaptation and resonances. The input transformation from current to somatic and then axonal voltage can be achieved by an AIS-soma-dendrite three-compartment model similar to the two-compartment model utilized by Ostojic and colleagues 15]. Such few-compartment models can also explicitly represent the electrotonic separation between soma and AIS, which shapes the signal arriving at the AP initiation zone 25, 26]. Alternatively, the electrotonic structure of the somato-dendritic compartments can be represented by analytical transformations, similar to the work of Aspart and colleagues 27. A combination of those approaches could mimic the dynamic gain curves of real neurons, their dependence on working points and even input correlations, all at much lower computational cost than a conductance-based, multi-compartmental neuron model.

Cellular physiology of dynamic population coding Because dynamic gain decomposition works along the biophysical signal transformation cascade, its results can be interpreted in physiological terms and corroborated with conventional electrophysiological methods, such as, potassium channel pharmacology [35]. This is particularly helpful in the emerging fields of axon initial segment plasticity and spectrinophaties, where structural changes at the site of AP initiation are observed as consequences of altered input ( $36-39$ or mutated structural molecules 9, 40, 41], and the consequences for excitability and encoding capacity need to be quantified in order to understand the functional impact on the population level. We propose that future studies of axon initial segment plasticity can use dynamic gain measurements at carefully maintained working points to quantify potential circuit-level consequences. Dynamic gain decomposition can then be used to inform mechanistic models of how the observed molecular and structural rearrangements affect functional differences or, alternatively, maintain encoding precision while changing excitability. Foundations for such connection between physiology and encoding precision have already been laid by studies that tie structural changes at

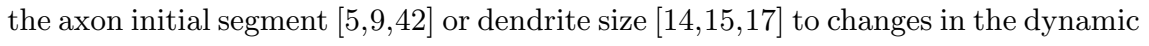
gain. It will be possible to apply the decomposition method to these recently obtained data, to attribute the observed changes to sub- and suprathreshold signal transformations, and to thereby critically test the concepts formulated in those studies. Decomposing dynamic gains from differently treated pyramidal neurons, we demonstrated which insights can be gained even from experimental data. First, it reveals the strong impact of the effective impedance. Second, it confirms the relation between the initiation current's voltage dependence and the bandwidth of the dynamic gain. Unlike Eyal's model, the real neuron's encoding bandwidth is limited by the initiation dynamics. This opens up new questions for future research, because the biophysical origin of the effective impedance's peculiar shape is unclear, as is the wide bandwidth of the initiation-related dynamic gain decay. The analysis of the experimental data also highlights the problem of choosing equivalent working points for different neurons. Ultimately, to understand population encoding in the brain, we need a better understanding of the physiological working points, experienced by neurons in vivo. Future experimental and theoretical studies will identify the biophysical basis for the surprisingly flexible dynamic gain changes at 
those working points [10, and illuminate how population encoding strategies can differ between brain states.

\section{References}

[1] Thorpe S, Fize D, Marlot C. Speed of processing in the human visual system. Nature. 1996;381(6582):520-522. doi:10.1038/381520a0.

[2] Stanford TR, Shankar S, Massoglia DP, Costello MG, Salinas E. Perceptual decision making in less than 30 milliseconds. Nat Neurosci. 2010;13(3):379-385. doi:10.1038/nn.2485.

[3] Tchumatchenko T, Malyshev A, Wolf F, Volgushev M. Ultrafast population encoding by cortical neurons. J Neurosci. 2011;31(34):12171-12179. doi:10.1523/JNEUROSCI.2182-11.2011.

[4] Knight BW. Dynamics of encoding in a population of neurons. J Gen Physiol. 1972;59(6):734-766. doi:10.1085/jgp.59.6.734.

[5] Revah O, Stoler O, Neef A, Wolf F, Fleidervish IA, Gutnick MJ. Dynamic gain analysis reveals encoding deficiencies in cortical neurons that recover from hypoxia-induced spreading depolarizations. J Neurosci. 2019;39(39):7790-7800. doi:10.1523/JNEUROSCI.3147-18.2019.

[6] Köndgen H, Geisler C, Fusi S, Wang XJ, Lüscher HR, Giugliano M. The dynamical response properties of neocortical neurons to temporally modulated noisy inputs in vitro. Cereb Cortex. 2008;18(September):2086-2097. doi:10.1093/cercor/bhm235.

[7] Boucsein C, Tetzlaff T, Meier R, Aertsen A, Naundorf B. Dynamical response properties of neocortical neuron ensembles: multiplicative versus additive noise. J Neurosci. 2009;29(4):1006-1010. doi:10.1523/JNEUROSCI.3424-08.2009.

[8] Higgs MH, Spain WJ. Conditional bursting enhances resonant firing in neocortical layer 2-3 pyramidal neurons. J Neurosci. 2009;29(5):1285-99. doi:10.1523/JNEUROSCI.3728-08.2009.

[9] Lazarov E, Dannemeyer M, Feulner B, Enderlein J, Gutnick MJ, Wolf $\mathrm{F}$, et al. An axon initial segment is required for temporal precision in action potential encoding by neuronal populations. Sci Adv. 2018;4(11). doi:10.1126/sciadv.aau8621.

[10] Merino RM, Leon-Pinzon C, Stühmer W, Möck M, Staiger JF, Wolf F, et al. Theta activity paradoxically boosts gamma and ripple frequency sensitivity in prefrontal interneurons. Proceedings of the National Academy of Sciences. 2021;118(51). doi:10.1073/pnas.2114549118.

[11] Eyal G, Mansvelder HD, de Kock CPJ, Segev I. Dendrites impact the encoding capabilities of the axon. J Neurosci. 2014;34(24):8063-71. doi:10.1523/JNEUROSCI.5431-13.2014.

[12] Broicher T, Malerba P, Dorval AD, Borisyuk A, Fernandez FR, White JA. Spike phase locking in CA1 pyramidal neurons depends on background conductance and firing rate. J Neurosci. 2012;32(41):14374-14388. doi:10.1523/JNEUROSCI.0842-12.2012.

[13] Ilin V, Malyshev A, Wolf F, Volgushev M. Fast computations in cortical ensembles require rapid initiation of action potentials. J Neurosci. 2013;33(6):228192. doi:10.1523/JNEUROSCI.0771-12.2013.

[14] Testa-Silva G, Verhoog MB, Linaro D, de Kock CPJ, Baayen JC, Meredith RM, et al. High bandwidth synaptic communication and frequency tracking in human neocortex. PLoS Biol. 2014;12(11):e1002007. doi:10.1371/journal.pbio.1002007. 
[15] Ostojic S, Szapiro G, Schwartz E, Barbour B, Brunel N, Hakim V. Neuronal morphology generates high-frequency firing resonance. J Neurosci. 2015;35(18):7056-7068. doi:10.1523/JNEUROSCI.3924-14.2015.

[16] Linaro D, Biró I, Giugliano M. Dynamical response properties of neocortical neurons to conductance-driven time-varying inputs. Eur J Neurosci. 2018;47(1):17-32. doi:10.1111/ejn.13761.

[17] Goriounova NA, Heyer DB, Wilbers R, Verhoog MB, Giugliano M, Verbist C, et al. Large and fast human pyramidal neurons associate with intelligence. eLife. 2018;7:1-21. doi:10.7554/eLife.41714.

[18] Borda Bossana S, Verbist C, Giugliano M. Homogeneous and Narrow Bandwidth of Spike Initiation in Rat L1 Cortical Interneurons. Frontiers in Cellular Neuroscience. 2020;14:118. doi:10.3389/fncel.2020.00118.

[19] Brunel N, Chance FS, Fourcaud N, Abbott LF. Effects of synaptic noise and filtering on the frequency response of spiking neurons. Phys Rev Lett. 2001;86(10):2186-2189. doi:10.1103/PhysRevLett.86.2186.

[20] Fourcaud-Trocmé N, Hansel D, van Vreeswijk C, Brunel N. How spike generation mechanisms determine the neuronal response to fluctuating inputs. J Neurosci. 2003;23(37):11628-11640. doi:23/37/11628 [pii].

[21] Naundorf B, Wolf F, Volgushev M. Unique features of action potential initiation in cortical neurons. Nature. 2006;440(April):1060-1063. doi:10.1038/nature04610.

[22] Wei W, Wolf F. Spike onset dynamics and response speed in neuronal populations. Phys Rev Lett. 2011;106(8):088102. doi:10.1103/PhysRevLett.106.088102.

[23] Huang M, Volgushev M, Wolf F. A small fraction of strongly cooperative sodium channels boosts neuronal encoding of high frequencies. PLoS ONE. 2012;7(5):e37629. doi:10.1371/journal.pone.0037629.

[24] Öz P, Huang M, Wolf F. Action potential initiation in a multi-compartmental model with cooperatively gating $\mathrm{Na}$ channels in the axon initial segment. J Comput Neurosci. 2015;39(1):63-75. doi:10.1007/s10827-015-0561-9.

[25] Brette R. Sharpness of spike initiation in neurons explained by compartmentalization. PLoS Comput Biol. 2013;9(12):e1003338. doi:10.1371/journal.pcbi.1003338.

[26] Zhang C, Hofmann D, Neef A, Wolf F. Ultrafast population coding and axosomatic compartmentalization. bioRxiv. 2021;doi:10.1101/2021.07.16.452655.

[27] Aspart F, Ladenbauer J, Obermayer K. Extending integrate-and-fire model neurons to account for the effects of weak electric fields and input filtering mediated by the dendrite. PLoS Comput Biol. 2016;12(11):1-29. doi:10.1371/journal.pcbi.1005206.

[28] Tchumatchenko T, Malyshev A, Geisel T, Volgushev M, Wolf F. Correlations and synchrony in threshold neuron models. Phys Rev Lett. 2010;104(5):1-10. doi:10.1103/PhysRevLett.104.058102.

[29] Vilela RD, Lindner B. Comparative study of different integrate-and-fire neurons: Spontaneous activity, dynamical response, and stimulus-induced correlation. Phys Rev E. 2009;80(3):1-12. doi:10.1103/PhysRevE.80.031909.

[30] Mainen ZF, Sejnowski TJ. Influence of dendritic structure on firing pattern in model neocortical neurons. Nature. 1996;382(6589):363-366. doi:10.1038/382363a0.

[31] Carnevale NT, Hines ML. The NEURON book. Cambridge University Press; 2006.

[32] Xiao-Jing Wang and György Buzsáki. Gamma oscillation by synaptic inhibition in a hippocampal interneuronal network model. J Neurosci. 1996;16(20):6402-6413. doi:10.1523/JNEUROSCI.16-20-06402.1996. 
[33] Lindner B, Schimansky-geier L. Transmission of noise coded versus additive signals through a neuronal ensemble. Phys Rev Lett. 2001;86(14):2934-2937. doi:10.1103/PhysRevLett.86.2934.

[34] Tchumatchenko T, Wolf F. Representation of dynamical stimuli in populations of threshold neurons. PLoS Comput Biol. 2011;7(10). doi:10.1371/journal.pcbi.1002239.

[35] Higgs MH, Spain WJ. Kv1 channels control spike threshold dynamics and spike timing in cortical pyramidal neurones. J Physiol. 2011;589(21):5125-5142. doi:10.1113/jphysiol.2011.216721.

[36] Grubb MS, Burrone J. Activity-dependent relocation of the axon initial segment fine-tunes neuronal excitability. Nature. 2010;465(7301):1070-1074. doi:10.1038/nature09160.

[37] Grubb MS, Shu Y, Kuba H, Rasband MN, Wimmer VC, Bender KJ. Short- and long-term plasticity at the axon initial segment. J Neurosci. 2011;31(45):1604916055. doi:10.1523/JNEUROSCI.4064-11.2011.

[38] Kuba H, Oichi Y, Ohmori H. Presynaptic activity regulates $\mathrm{Na}(+)$ channel distribution at the axon initial segment. Nature. 2010;465(7301):1075-8. doi:10.1038/nature09087.

[39] Jamann N, Dannehl D, Lehmann N, Wagener R, Thielemann C, Schultz C, et al. Sensory input drives rapid homeostatic scaling of the axon initial segment in mouse barrel cortex. Nature Communications. 2021;12(1):23. doi:10.1038/s41467-020-20232-x.

[40] Parkinson NJ, Olsson CL, Hallows JL, McKee-Johnson J, Keogh BP, NobenTrauth K, et al. Mutant $\beta$-spectrin 4 causes auditory and motor neuropathies in quivering mice. Nature Genetics. 2001;29(1):61-65. doi:10.1038/ng710.

[41] Wang CC, Ortiz-González XR, Yum SW, Gill SM, White A, Kelter E, et al. $\beta$-IV Spectrinopathies Cause Profound Intellectual Disability, Congenital Hypotonia, and Motor Axonal Neuropathy. The American Journal of Human Genetics. 2018;102(6):1158-1168. doi:10.1016/j.ajhg.2018.04.012.

[42] Nikitin ES, Bal NV, Malyshev A, Ierusalimsky VN, Spivak Y, Balaban PM, et al. Encoding of high frequencies improves with maturation of action potential generation in cultured neocortical neurons. Front Cell Neurosci. 2017;11(February):1-12. doi:10.3389/fncel.2017.00028. 